Research Article

\title{
Nonlinear Vibrations of Laminated Cross-Ply Composite Cantilever Plate in Subsonic Air Flow
}

\author{
Gen Liu, Wei Zhang $(\mathbb{D}$, and An Xi \\ Beijing Key Laboratory of Nonlinear Vibrations and Strength of Mechanical Structures College of Mechanical Engineering, \\ Beijing University of Technology, Beijing 100124, China \\ Correspondence should be addressed to Wei Zhang; sandyzhang0@yahoo.com
}

Received 31 July 2019; Revised 27 December 2019; Accepted 28 February 2020; Published 22 April 2020

Academic Editor: Fotios Georgiades

Copyright $(92020$ Gen Liu et al. This is an open access article distributed under the Creative Commons Attribution License, which permits unrestricted use, distribution, and reproduction in any medium, provided the original work is properly cited.

The nonlinear vibrations and responses of a laminated composite cantilever plate under the subsonic air flow are investigated in this paper. The subsonic air flow around the three-dimensional cantilever rectangle laminated composite plate is considered to be decreasing from the wing root to the wing tip. According to the ideal incompressible fluid flow condition and the Kutta-Joukowski lift theorem, the subsonic aerodynamic lift on the three-dimensional finite length flat wing is calculated by using the Vortex Lattice (VL) method. The finite length flat wing is modeled as a laminated composite cantilever plate based on Reddy's third-order shear deformation plate theory and the von Karman geometry nonlinearity is introduced. The nonlinear partial differential governing equations of motion for the laminated composite cantilever plate subjected to the subsonic aerodynamic force are established via Hamilton's principle. The Galerkin method is used to separate the partial differential equations into two nonlinear ordinary differential equations, and the four-dimensional nonlinear averaged equations are obtained by the multiple scale method. Through comparing the natural frequencies of the linear system with different material and geometric parameters, the relationship of $1: 2$ internal resonance is considered. Corresponding to several selected parameters, the frequency-response curves are obtained. The hardening-spring-type behaviors and jump phenomena are exhibited. The influence of the force excitation on the bifurcations and chaotic behaviors of the laminated composite cantilever plate is investigated numerically. It is found that the system is sensitive to the exciting force according to the complicate nonlinear behaviors exhibited in this paper.

\section{Introduction}

The vibration of the plate and shell structures owing to air flow is a matter of interest because of its significance in design of launch vehicles and aircrafts [1]. Laminated composite structures are widely used in aerospace field due to its high strength-to-weight ratio, light weight, and long fatigue life. The dynamic behavior of the laminated composite plates in air flow has gained significant focus of attention for many researchers. However, there are few research works dealing with the complex nonlinear dynamics of the structure which is simplified as a laminated composite cantilever plate subjected to subsonic air flow. Therefore, the nonlinear dynamics of laminated composite plates in subsonic flow will be worth analyzing in this work.

In the 1990s, the research on the vibration of plates has been studied comprehensively. Some literature reviews on nonlinear vibrations of plates were given by Chia $[2,3]$ and Mehar and Panda [4]. The nonlinear vibrations of laminated composite spherical shell panels were also entirely investigated by Mahapatra et al. [5-9]. The vibration, bending, and buckling behaviors about the functionally graded sandwich structure have been investigated by Mehar et al. [10-13] and Kar and Panda [14-16]. A third-order theory which accounted for a cubic variation of the in-plane displacements through the plate thickness was derived by Librescu and Reddy [17]. Great progress has also been achieved in the study of the nonlinear thermoelastic frequency analysis [18-22]. Zhang [23] studied the global bifurcations and chaotic dynamics of simply supported rectangular thin plates under parametrical excitation by introducing von Karman's geometric nonlinearity plate theory.

Nonlinear analysis considering the geometrical and material nonlinearity by FEM was conducted by Panda and 
Singh [24-26]. Onkar and Yadav [27] studied the nonlinear random vibrations of a simply supported cross-ply laminated composite plate using analytical methods, and the accuracy of response evaluation was improved in this work. Park et al. [28] investigated the nonlinear forced vibrations of skew sandwich plates subjected to multiple of dynamic loads, the influence of the skew angles, boundary conditions, and loads on the nonlinear dynamic behaviors of composite plates which were discussed. Chien and Chen [29] studied the effects of initial stresses and different parameters on the nonlinear vibrations of laminated composite plates on elastic foundations. Singh et al. [30] used the higher-order shear deformation plate theory to study the dynamic responses of a geometrically nonlinear laminated composite plate lying on different elastic foundations. Zhang et al. [31, 32] investigated nonlinear vibration, bifurcation, and chaotic dynamics of laminated composite plates. Alijani et al. $[33,34]$ studied nonlinear vibrations of FGM plates and shallow shells, and the bifurcations and nonlinear dynamic behaviors were analyzed in their papers. Amabili [35] used the higher-order shear deformation theory to study the nonlinear vibrations of angle-ply laminated circular cylindrical shells. Most recently, Zhang et al. [36-39] studied the nonlinear vibrations of the laminated circular cylindrical shells based on the deployed ring truss for antenna structures. The complex nonlinear dynamics of the circular cylindrical shell were studied and the bifurcations and chaotic behaviors were investigated by using the analytical methods and numerical simulations.

The research on nonlinear vibrations of plates and shells excited by the aerodynamic force were also widely investigated by researchers. In the early years, Dowell $[40,41]$ studied the nonlinear oscillations of the fluttering plate systematically. Literature reviews on the nonlinear vibrations of circular cylindrical shells and panels with and without fluid-structure interaction were given by Amabili and Par"doussis [42]. The studies on the nonlinear vibration of laminated plates in air flow were mostly about the plates in the supersoninc flow. Singha and Ganapathi [43] investigated the effect of the system parameters on supersonic panel flutter behaviors of laminated composite plates. Haddadpour et al. [44] investigated the nonlinear aeroelastic behaviors of FGM. Singha and Mandal [45] used a 16-node isoparametric degenerated shell element to study the supersonic panel flutter behaviors of laminated composite plates and cylindrical panels. Kuo [46] investigated the effect of variable fiber spacing on the supersonic flutter of rectangular composite plates. Zhao and Zhang [47] presented the analysis of the nonlinear dynamics for a laminated composite cantilever rectangular plate subjected to the supersonic gas flows and the in-plane excitations. An analysis on the nonlinear dynamics of an FGM plate in hypersonic flow subjected to an external excitation and uniform temperature change was presented by Hao et al. [48]. The nonlinear dynamic behavior of an axially extendable cantilever laminated composite plate using piezoelectric materials under the combined action of aerodynamic load and piezoelectric excitation was studied by Lu et al. [49]. Yao and Li [50] investigated the nonlinear vibration of a two-dimensional laminated composite plate in subsonic air flow with simply supported boundary conditions. A simple subsonic aerodynamics model was introduced in this work, which was used to analyze twodimensional infinite length plate.

In this paper, the nonlinear dynamics of the laminated composite cantilever plate under subsonic air flow were investigated. According to the flow condition of ideal incompressible fluid and the Kutta-Joukowski lift theorem, the subsonic aerodynamic lift on the three-dimensional finite length flat wing was calculated using the Vortex Lattice (VL) method. The nonlinear partial differential governing equations of motion for the laminated composite cantilever plate subjected to the subsonic aerodynamic force were established via Hamilton's principle. The Galerkin method was used to separate the partial differential equation into two nonlinear ordinary differential equations. The numerical method was utilized to investigate the bifurcations and periodic and chaotic motions of the composite laminated rectangular plate. The numerical results illustrate that there existed the periodic and chaotic motions of the composite laminated cantilever plate. The hardening-spring characteristics of the composite laminated cantilever plate were demonstrated by the frequencyresponse curves of the system.

\section{Derivation of the Subsonic Aerodynamic Force on the Plate}

2.1. Vortex Lattice (VL) Method. In this section, the subsonic air flow on the plate will be calculated by using the VL method. The VL method is a numerical implementation on the general 3D lifting surface problem. This method discretizes the vortex-sheet strength distributing on each lifting surface by lumping it into a collection of horseshoe vortices. Figure 1 shows that a $3 \mathrm{D}$ lifting surface is discretized by a vortex lattice of horseshoe vortices, in which all contribute to the velocity $V$ at any field point $r$.

For the finite wingspan, the high-pressure air below the wing will turn over the low pressure air at the tip of the wing, which will cause the pressure on the upper surface of the wing tip to be equal to that of the lower surface. Unlike the two-dimensional flow around the airfoil, the main characteristic of the three-dimensional flow around the wing is the variation of the lift along the wingspan. In order to calculate the lift on the wing surface by using the vortex lattice method, the Biot-Savart law is used to calculate the induced velocity on the control point. The vortex strength of the vortex system is obtained, and the pressure difference on the upper and lower surface of the wing surface is deduced at the same time.

The velocity induced by a vortex line with a strength of $\Gamma_{n}$ and a length of $\mathrm{d} l$ is calculated by the Biot-Savart law as

$$
\mathrm{d} \mathbf{V}=\frac{\Gamma_{n}(\mathrm{~d} \mathbf{l} \times \mathbf{r})}{4 \pi r^{3}}
$$

As shown in Figure 2, the induced velocity is

$$
d V=\frac{\Gamma_{n} \sin \theta \mathrm{d} l}{4 \pi r^{2}} .
$$




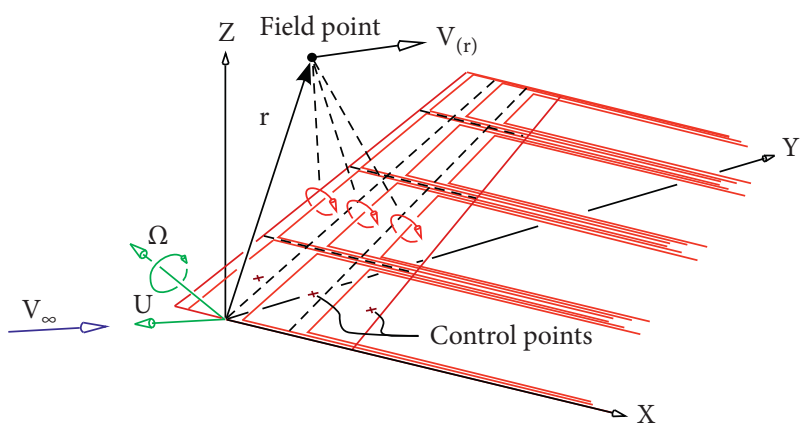

Figure 1: A 3D lifting surface discretized by a vortex lattice of horseshoe vortices.

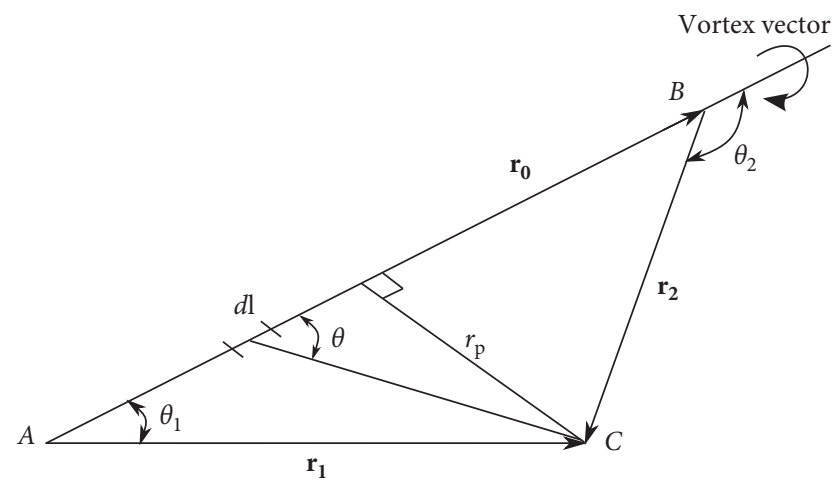

Figure 2: The velocity induced by finite length vortex segments.

Let $A B$ represent a vortex segment in which the vortex vector points from $A$ to $B$. $C$ is a space point, and its normal distance from the $A B$ line is $r_{p}$.

We can make an integral from the point $A$ to the point $B$ to find out the size of the induced velocity:

$$
V=\frac{\Gamma_{n}}{4 \pi r_{p}} \int_{\theta_{1}}^{\theta_{2}} \sin \theta \mathrm{d} \theta=\frac{\Gamma_{n}}{4 \pi r_{p}}\left(\cos \theta_{1}-\cos \theta_{2}\right) .
$$

As $\mathbf{r}_{0}, \mathbf{r}_{1}$, and $\mathbf{r}_{2}$, respectively, represent the $\mathbf{A B}, \mathbf{A C}$, and $\mathbf{B C}$ in Figure 2, the following relationships are established:

$$
\begin{aligned}
r_{p} & =\frac{\left|\mathbf{r}_{1} \times \mathbf{r}_{2}\right|}{r_{0}}, \\
\cos \theta_{1} & =\frac{\mathbf{r}_{0} \cdot \mathbf{r}_{1}}{\mathbf{r}_{0} \mathbf{r}_{1}}, \\
\cos \theta_{2} & =\frac{\mathbf{r}_{0} \cdot \mathbf{r}_{2}}{\mathbf{r}_{0} \mathbf{r}_{2}} .
\end{aligned}
$$

Then, the formula calculating the induced velocity generated by the horseshoe vortex in the vortex lattice method is derived:

$$
\mathbf{V}=\frac{\Gamma_{n}}{4 \pi} \frac{\mathbf{r}_{1} \times \mathbf{r}_{2}}{\left|\mathbf{r}_{1} \times \mathbf{r}_{2}\right|}\left[\mathbf{r}_{0} \cdot\left(\frac{\mathbf{r}_{1}}{r_{1}}-\frac{\mathbf{r}_{2}}{r_{2}}\right)\right] .
$$

From equation (5), the velocity induced by a vortex segment $A B$ at any point in the coordinate $(x, y, z)$ can be obtained:

$$
\begin{aligned}
\mathbf{V}_{\mathbf{A B}}= & \frac{\Gamma_{n}}{4 \pi}\left\{\text { Fac1 }_{A B}\right\} \cdot\left\{F a c 2_{A B}\right\}, \\
\mathbf{V}_{\mathbf{A} \infty}= & \frac{\Gamma_{n}}{4 \pi}\left\{\frac{\left(z-z_{1 n}\right) \mathbf{j}+\left(y-y_{1 n}\right) \mathbf{k}}{\left[\left(z-z_{1 n}\right)^{2}+\left(y-y_{1 n}\right)^{2}\right]}\right\} \\
& \times\left[1.0+\frac{x-x_{1 n}}{\left(x-x_{1 n}\right)^{2}+\left(y-y_{1 n}\right)^{2}+\left(z-z_{1 n}\right)^{2}}\right], \\
\mathbf{V}_{\mathbf{B} \infty}= & -\frac{\Gamma_{n}}{4 \pi}\left\{\frac{\left(z-z_{2 n}\right) \mathbf{j}+\left(y_{1 n}-y\right) \mathbf{k}}{\left[\left(z-z_{1 n}\right)^{2}+\left(y_{2 n}-y\right)^{2}\right]}\right\} \\
& \times\left[1.0+\frac{x-x_{2 n}}{\left(x-x_{2 n}\right)^{2}+\left(y-y_{2 n}\right)^{2}+\left(z-z_{2 n}\right)^{2}}\right],
\end{aligned}
$$

where $\mathbf{V}_{\mathrm{A} \infty}$ is the velocity induced by vortex lines from the point $A$ to $\infty$ along the $x$-axis and $\mathbf{V}_{\mathrm{B} \infty}$ is the velocity induced by vortex lines from the point $B$ to $\infty$ along the $x$-axis.

The total velocity induced by a horseshoe vortex at a point $(x, y, z)$ representing a surface element (i.e., the $n$th panel element) is the sum of the various components calculated by equations $(6 a)-(6 c)$. Therefore, the velocity induced by $2 \mathrm{~N}$ vortexes is obtained to get the total induced velocity on the first $m$ control points. It is expressed as

$$
\mathbf{V}_{\mathbf{m}}=\sum_{n=1}^{2 N} \mathbf{C}_{\mathbf{m}, \mathbf{n}} \Gamma_{n}
$$

The contribution of all vortices to the downwash of the control point for the $m$ th panel element:

$$
w_{m}=\sum_{n=1}^{2 N} w_{m, n} .
$$

Then, the lift on the rectangular cantilever plate with a sweep rectangular wing surface is computed according to the VL method. The vortex system is arranged on the rectangular wing surface in Figure 3.

2.2. Application of VL Method on the Plate. In Figure 3, the variable $b$ is twice the span of the wing, $c$ is the taper ratio of the wing surface, and $c$ is equal to 1 . The sweep angle is $0^{\circ}$. Five panel elements are specified on the wing surface, and the vortex line is arranged at the leading edge $1 / 4$ chord, and the control point is located at $3 / 4$ chord. The wing surface is divided into 5 panel elements and each panel extends from the leading edge to the trailing edge.

The coordinates of the $5 \times 1$ vortices on the wing surface are listed in Table 1.

The downwash velocity of each surface element induced at the control point is superimposed through the nonpenetrating condition:

$$
w_{m}=-V_{\infty} \alpha .
$$




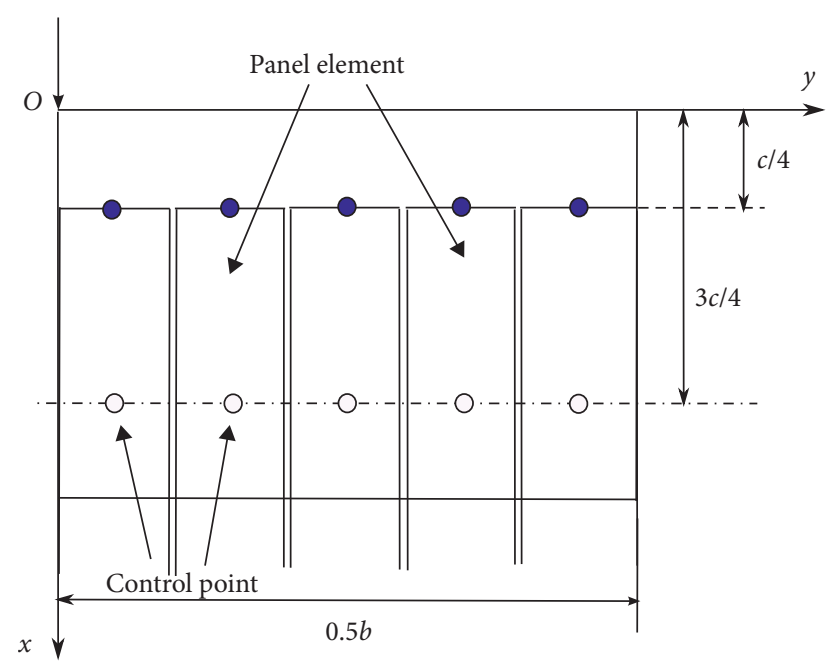

Figure 3: Layout of the vortex lattice on the flat wing surface.

TABLE 1: The coordinates of the attachment vortex and the control point in the wing surface.

\begin{tabular}{lcccccc}
\hline $\begin{array}{l}\text { Panel element } \\
\text { number }\end{array}$ & $x_{m}$ & $y_{m}$ & $x_{1 n}$ & $y_{1 n}$ & $x_{2 n}$ & $y_{2 n}$ \\
\hline 1 & $0.25 b$ & $0.05 b$ & $0.833 b$ & $0 b$ & $0.833 b$ & $0.1 b$ \\
2 & $0.25 b$ & $0.15 b$ & $0.833 b$ & $0.1 b$ & $0.833 b$ & $0.2 b$ \\
3 & $0.25 b$ & $0.25 b$ & $0.833 b$ & $0.2 b$ & $0.833 b$ & $0.15 b$ \\
4 & $0.25 b$ & $0.35 b$ & $0.833 b$ & $0.15 b$ & $0.833 b$ & $0.4 b$ \\
5 & $0.25 b$ & $0.45 b$ & $0.833 b$ & $0.4 b$ & $0.833 b$ & $0.5 b$ \\
\hline
\end{tabular}

The solution of vortex strength is obtained by using 5 algebraic equations with unknown vortex strength:

$$
\begin{aligned}
& \Gamma_{1}=0.10082\left(4 \pi b V_{\infty} \alpha\right), \\
& \Gamma_{2}=0.13180\left(4 \pi b V_{\infty} \alpha\right), \\
& \Gamma_{3}=0.13901\left(4 \pi b V_{\infty} \alpha\right), \\
& \Gamma_{4}=0.12496\left(4 \pi b V_{\infty} \alpha\right), \\
& \Gamma_{5}=0.09887\left(4 \pi b V_{\infty} \alpha\right) .
\end{aligned}
$$

According to the boundary conditions, the air flow is tangent to the object surface at each control point to determine the strength of each vortex. The lift of the wing can be calculated by satisfying the boundary conditions. For any wing that does not have an upper counter angle, the lift is produced by the free flow that crosses the vortex line as there is no side-washing speed or postwash speed.

According to a finite number of elements, the lift on the plate is expressed as

$$
L_{0}=\rho_{\infty} V_{\infty} \sum_{n=1}^{5} \Gamma_{n} \Delta y_{n}
$$

As the geometric relationship of each facet is $\Delta y_{n}=0.1 b$, the lift on the flat plate can be rewritten as

$$
L_{0}=\rho_{\infty} V_{\infty}^{2} \pi b^{2} \alpha(0.238184)
$$

where $\rho_{\infty}$ is the flow density, $V_{\infty}$ is the flow velocity, $b$ is half length of the wingspan, and $\alpha$ is the attack angle.

The attack angle is considered to be affected by a periodic disturbance $\alpha=\alpha_{0}+\alpha_{1} \cos \Omega_{2} t$. Taking the periodic perturbation into the aerodynamic force and according to equation (12), the expression of the aerodynamic force containing the perturbation term is obtained:

$$
L=L_{0}+L_{1} \cos \Omega_{2} t,
$$

where $\quad L_{0}=\rho_{\infty} V_{\infty}^{2} \pi b^{2} \alpha_{0}(0.238184)$

and

\section{Formulation}

In this section, the dynamic equations of the laminated composite cantilever plate are derived. As shown in Figure 4, the parameters $x, y$, and $z$ are, respectively, the spanwise direction, the direction of the chord, and the vertical direction of the plate. The plate is clamped at the position $x=0$. The ply stacking sequence is $\left[0^{\circ} / 90^{\circ}\right]_{S}$ and the layer number is $N$. The in-plane excitation is $F=F_{0}+F_{1} \cos \Omega_{1} t$, which is distributed along the chord direction of the plate. The vertical direction of the plate along the spanwise direction is subject to subsonic aerodynamic force $L$.

The nonlinear governing equations are established in the Cartesian coordinate system. Reddy's third-order shear deformation plate theory is used:

$$
u(x, y, t)=u_{0}(x(t), y, t)+z \phi_{x}(x(t), y, t)-z^{3} \frac{4}{3 h^{2}}\left(\phi_{x}+\frac{\partial w_{0}}{\partial x}\right),
$$

$$
v(x, y, t)=v_{0}(x(t), y, t)+z \phi_{y}(x(t), y, t)-z^{3} \frac{4}{3 h^{2}}\left(\phi_{y}+\frac{\partial w_{0}}{\partial y}\right),
$$

$w(x, y, t)=w_{0}(x(t), y, t)$.

The von Karman nonlinear strain-displacement relation is introduced. The displacements and strain-displacement relation are given as follows:

$$
\begin{aligned}
& \varepsilon_{x x}=\frac{\partial u_{0}}{\partial x}+\frac{1}{2}\left(\frac{\partial w_{0}}{\partial x}\right)^{2}, \\
& \varepsilon_{y y}=\frac{\partial v_{0}}{\partial y}+\frac{1}{2}\left(\frac{\partial w_{0}}{\partial y}\right)^{2}, \\
& \gamma_{x y}=\frac{1}{2}\left(\frac{\partial u_{0}}{\partial y}+\frac{\partial v_{0}}{\partial x}+\frac{\partial w_{0}}{\partial x} \frac{\partial w_{0}}{\partial y}\right), \\
& \gamma_{y z}=\frac{1}{2}\left(\frac{\partial v_{0}}{\partial z}+\frac{\partial w_{0}}{\partial y}\right), \\
& \gamma_{z x}=\frac{1}{2}\left(\frac{\partial u_{0}}{\partial z}+\frac{\partial w_{0}}{\partial x}\right) .
\end{aligned}
$$




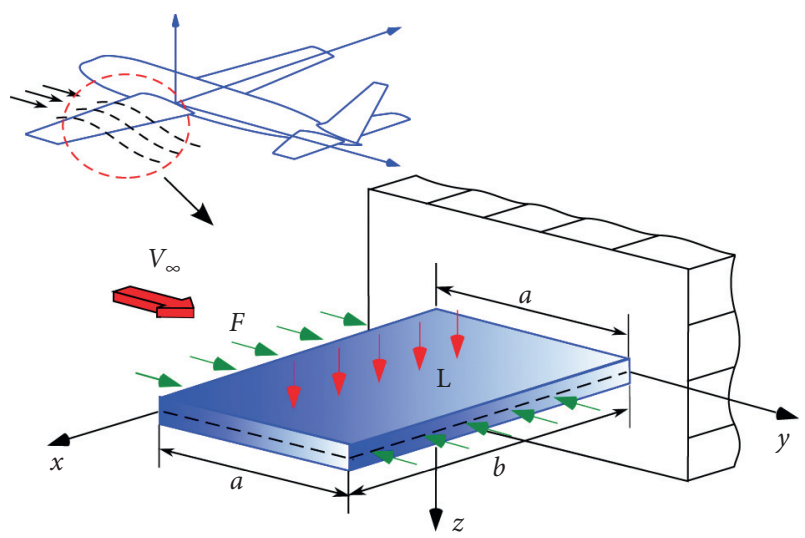

FIgURE 4: Mechanical model of cantilever laminated composite plate.

Substituting the expressions of the strain into the displacements, we can obtain

$$
\begin{aligned}
\left\{\begin{array}{c}
\varepsilon_{x x} \\
\varepsilon_{y y} \\
\gamma_{x y}
\end{array}\right\} & =\left\{\begin{array}{c}
\varepsilon_{x}^{(0)} \\
\varepsilon_{y}^{(0)} \\
\gamma_{x y}^{(0)}
\end{array}\right\}+z\left\{\begin{array}{c}
\varepsilon_{x}^{(1)} \\
\varepsilon_{y}^{(1)} \\
\gamma_{x y}^{(1)}
\end{array}\right\}+z^{3}\left\{\begin{array}{c}
\varepsilon_{x}^{(3)} \\
\varepsilon_{y}^{(3)} \\
\gamma_{x y}^{(3)}
\end{array}\right\}, \\
\left\{\begin{array}{l}
\gamma_{y z} \\
\gamma_{z x}
\end{array}\right\} & =\left\{\begin{array}{l}
\gamma_{y z}^{(0)} \\
\gamma_{z x}^{(0)}
\end{array}\right\}+z^{2}\left\{\begin{array}{l}
\gamma_{y z}^{(2)} \\
\gamma_{z x}^{(2)}
\end{array}\right\},
\end{aligned}
$$

where

$$
\begin{aligned}
& \left\{\begin{array}{c}
\varepsilon_{x}^{(0)} \\
\varepsilon_{y}^{(0)} \\
\gamma_{x y}^{(0)}
\end{array}\right\}=\left\{\begin{array}{c}
\frac{\partial u_{0}}{\partial x}+\frac{1}{2}\left(\frac{\partial w_{0}}{\partial x}\right)^{2} \\
\frac{\partial v_{0}}{\partial y}+\frac{1}{2}\left(\frac{\partial w_{0}}{\partial y}\right)^{2} \\
\frac{\partial u_{0}}{\partial y}+\frac{\partial v_{0}}{\partial x}+\frac{\partial w_{0}}{\partial x} \frac{\partial w_{0}}{\partial y}
\end{array}\right\}\left\{\begin{array}{c}
\varepsilon_{x x}^{(1)} \\
\varepsilon_{y y}^{(1)} \\
\gamma_{x y}^{(1)}
\end{array}\right\}=\left\{\begin{array}{c}
\frac{\partial \phi_{x}}{\partial x} \\
\frac{\partial \phi_{y}}{\partial y} \\
\frac{\partial \phi_{x}}{\partial y}+\frac{\partial \phi_{y}}{\partial x}
\end{array}\right\}, \\
& \left\{\begin{array}{c}
\varepsilon_{x x}^{(3)} \\
\varepsilon_{y y}^{(3)} \\
\gamma_{x y}^{(3)}
\end{array}\right\}=-c_{1}\left\{\begin{array}{c}
\frac{\partial \phi_{x}}{\partial x}+\frac{\partial^{2} w_{0}}{\partial x^{2}} \\
\frac{\partial \phi_{y}}{\partial y}+\frac{\partial^{2} w_{0}}{\partial y^{2}} \\
\frac{\partial \phi_{x}}{\partial y}+\frac{\partial \phi_{y}}{\partial x}+2 \frac{\partial^{2} w_{0}}{\partial x \partial y}
\end{array}\right\} \\
& \left\{\begin{array}{l}
\gamma_{y z}^{(0)} \\
\gamma_{z x}^{(0)}
\end{array}\right\}=\left\{\begin{array}{c}
\phi_{y}+\frac{\partial w_{0}}{\partial y} \\
\phi_{x}+\frac{\partial w_{0}}{\partial x}
\end{array}\right\}, \\
& \left\{\begin{array}{c}
\gamma_{y z}^{(2)} \\
\gamma_{z x}^{(2)}
\end{array}\right\}=-c_{2}\left\{\begin{array}{c}
\phi_{y}+\frac{\partial w_{0}}{\partial y} \\
\phi_{x}+\frac{\partial w_{0}}{\partial x}
\end{array}\right\},
\end{aligned}
$$

where

$$
\begin{aligned}
& c_{1}=\frac{4}{3 h^{2}}, \\
& c_{2}=3 c_{1} .
\end{aligned}
$$

The symmetric cross-ply laminated composite plate is adopted, and we can obtain

$$
\left\{\begin{array}{c}
\sigma_{x x} \\
\sigma_{y y} \\
\sigma_{y z} \\
\sigma_{z x} \\
\sigma_{x y}
\end{array}\right\}=\left\{\begin{array}{ccccc}
\bar{Q}_{11} & \bar{Q}_{12} & 0 & 0 & 0 \\
\bar{Q}_{21} & \bar{Q}_{22} & 0 & 0 & 0 \\
0 & 0 & \bar{Q}_{44} & 0 & 0 \\
0 & 0 & 0 & \bar{Q}_{55} & 0 \\
0 & 0 & 0 & 0 & \bar{Q}_{66}
\end{array}\right\}\left\{\begin{array}{c}
\varepsilon_{x x} \\
\varepsilon_{y y} \\
\gamma_{y z} \\
\gamma_{z x} \\
\gamma_{x y}
\end{array}\right\},
$$

where

$$
\left\{\begin{array}{c}
\bar{Q}_{11} \\
\bar{Q}_{12} \\
\bar{Q}_{22} \\
\bar{Q}_{16} \\
\bar{Q}_{26} \\
\bar{Q}_{66}
\end{array}\right\}=\left\{\begin{array}{cccc}
C^{4} & 2 C^{2} S^{2} & S^{2} & 4 C^{2} S^{2} \\
C^{2} S^{2} & C^{4}+S^{4} & C^{2} S^{2} & -4 C^{2} S^{2} \\
S^{4} & 2 C^{2} S^{2} & C^{4} & 4 C^{2} S^{2} \\
C^{3} S & C S^{3}-C^{3} S & -C S^{3} & -2 C S\left(C^{2}-S^{2}\right) \\
C S^{3} & C^{3} S-C S^{3} & -C^{3} S & 2 C S\left(C^{2}-S^{2}\right) \\
C^{2} S^{2} & -2 C^{2} S^{2} & C^{2} S^{2} & \left(C^{2}-S^{2}\right)^{2}
\end{array}\right\}\left\{\begin{array}{c}
Q_{11} \\
Q_{12} \\
Q_{22} \\
Q_{66}
\end{array}\right\},
$$

$$
\begin{aligned}
\left\{\begin{array}{l}
\bar{Q}_{44} \\
\bar{Q}_{45} \\
\bar{Q}_{55}
\end{array}\right\} & =\left\{\begin{array}{cc}
C^{2} & S^{2} \\
-C S & C S \\
S^{2} & C^{2}
\end{array}\right\}\left\{\begin{array}{l}
Q_{44} \\
Q_{55}
\end{array}\right\}, \\
C & =\cos \theta, \\
S & =\sin \theta,
\end{aligned}
$$

where $\theta$ is the angle of laminated layer. The stiffness of each layer is

$$
\begin{aligned}
Q_{11} & =\frac{E_{1}}{1-\nu_{12} \nu_{21}}, \\
Q_{12} & =\frac{\nu_{12} E_{2}}{1-\nu_{12} \nu_{21}}, \\
Q_{22} & =\frac{E_{2}}{1-\nu_{12} \nu_{21}}, \\
Q_{44} & =Q_{55}=Q_{66}=G_{12},
\end{aligned}
$$

where $E_{i}$ is the Young modulus, $G_{12}$ is the shear modulus, and $\nu_{12}$ is Poisson's ratio.

According to Hamilton's principle,

$$
\int_{0}^{T}(\delta K-\delta U+\delta W) \mathrm{d} t=0 .
$$

The nonlinear governing equations of motion are given as follows: 


$$
\begin{aligned}
& N_{x x, x}+N_{x y, y}=I_{0} \ddot{u}_{0}+\left(I_{1}-c_{1} I_{3}\right) \ddot{\varphi}_{x}-c_{1} I_{3} \frac{\partial \ddot{w}_{0}}{\partial x}, \quad(25 \mathrm{a}) \\
& N_{y y, y}+N_{x y, x}=I_{0} \ddot{v}_{0}+\left(I_{1}-c_{1} I_{3}\right) \ddot{\varphi}_{y}-c_{1} I_{3} \frac{\partial \ddot{w}_{0}}{\partial y}, \quad(25 \mathrm{~b}) \\
& N_{y y, y} \frac{\partial w_{0}}{\partial y}+N_{y y} \frac{\partial^{2} w_{0}}{\partial y^{2}}+N_{x y, x} \frac{\partial w_{0}}{\partial y}+N_{x y, y} \frac{\partial w_{0}}{\partial x}+N_{x x, x} \frac{\partial w_{0}}{\partial x} \\
& +N_{x x} \frac{\partial^{2} w_{0}}{\partial x^{2}}+c_{1}\left(P_{x x, x x}+2 P_{x y, x y}+P_{y y, y y}\right)+2 N_{x y} \frac{\partial^{2} w_{0}}{\partial x \partial y} \\
& +\left(Q_{x, x}-c_{2} R_{x, x}\right) \\
& +\left(Q_{y, y}-c_{2} R_{y, y}\right)+L-\gamma \dot{w}_{0}=c_{1} I_{3}\left(\frac{\partial \ddot{u}_{0}}{\partial x}+\frac{\partial \ddot{v}}{\partial y}\right) \\
& +c_{1}\left(I_{4}-c_{1} I_{6}\right)\left(\frac{\partial \ddot{\varphi}_{x}}{\partial x}+\frac{\partial \ddot{\varphi}_{y}}{\partial y}\right)+I_{0} \ddot{w}_{0}-c_{1}^{2} I_{6}\left(\frac{\partial^{2} \ddot{w}_{0}}{\partial x^{2}}+\frac{\partial^{2} \ddot{w}_{0}}{\partial y^{2}}\right), \\
& \quad+\left(I_{1}-c_{1} I_{3}\right) \ddot{v}_{0}+\left(I_{2}-2 c_{1} I_{4}+c_{1}^{2} I_{6}\right) \ddot{\varphi}_{y}-c_{1}\left(I_{4}-c_{1} I_{6}\right) \frac{\partial \ddot{w}_{0}}{\partial y}, \\
& M_{x x, x}+M_{x y, y}-c_{1} P_{x x, x}-c_{1} P_{x y, y}-\left(Q_{x}-c_{2} R_{x}\right) \\
& \quad=\left(I_{1}-c_{1} I_{3}\right) \ddot{u}_{0}+\left(I_{2}-2 c_{1} I_{4}+c_{1}^{2} I_{6}\right) \ddot{\varphi}_{x}-c_{1}\left(I_{4}-c_{1} I_{6}\right) \frac{\partial \ddot{w}_{0}}{\partial x},
\end{aligned}
$$

where $\gamma$ is the damping coefficient.

The internal force is expressed as follows:

$$
\begin{aligned}
& \left\{\begin{array}{c}
N_{\alpha \beta} \\
M_{\alpha \beta} \\
P_{\alpha \beta}
\end{array}\right\}=\int_{-(h / 2)}^{(h / 2)} \sigma_{\alpha \beta}\left\{\begin{array}{c}
1 \\
z \\
z^{3}
\end{array}\right\} d z, \\
& \left\{\begin{array}{c}
Q_{\alpha} \\
R_{\alpha}
\end{array}\right\}=\int_{-(h / 2)}^{(h / 2)} \sigma_{\alpha z}\left\{\begin{array}{c}
1 \\
z^{2}
\end{array}\right\} d z .
\end{aligned}
$$

Substituting the internal force of equation (26) into equations (25a)-(25e), we can obtain the expression of the governing equation of motion by the displacements, which are given in Appendix.

The boundary conditions of the cantilever plate are obtained at the same time as

$$
\begin{gathered}
x=0: w=v=u=\varphi_{y}=\varphi_{x}=0, \\
x=a: N_{x x}=N_{x y}=M_{x x}=M_{x y}-c_{1} P_{x y}=\bar{Q}_{x}=0, \\
y=0: N_{x y}=N_{y y}=M_{y y}=M_{x y}-c_{1} P_{x y}=\bar{Q}_{y}=0, \\
y=b: N_{x y}=N_{y y}=M_{y y}=M_{x y}-c_{1} P_{x y}=\bar{Q}_{y}=0,
\end{gathered}
$$

$$
\begin{aligned}
& \bar{Q}_{x}=Q_{x}+\frac{\partial M_{x y}}{\partial y}-c_{2} R_{x}+c_{1}\left(\frac{\partial P_{x x}}{\partial x}+\frac{\partial P_{x y}}{\partial y}\right), \\
& \bar{Q}_{y}=Q_{y}+\frac{\partial M_{x y}}{\partial x}-c_{2} R_{y}+c_{1}\left(\frac{\partial P_{y y}}{\partial y}+\frac{\partial P_{x y}}{\partial x}\right) .
\end{aligned}
$$

The dimensionless equations can be obtained by introducing the following parameters:

$$
\begin{aligned}
& \bar{w}=\frac{w_{0}}{h}, \\
& \bar{t}=t \pi^{2}\left(\frac{E}{a b \rho}\right)^{(1 / 2)}, \\
& \bar{A}_{i j}=\frac{A_{i j}(a b)^{(1 / 2)}}{E h^{2}} \text {, } \\
& \bar{B}_{i j}=\frac{(a b)^{(1 / 2)}}{E h^{3}} B_{i j}, \\
& \bar{D}_{i j}=\frac{(a b)^{(1 / 2)}}{E h^{4}} D_{i j}, \\
& \bar{E}_{i j}=\frac{(a b)^{(1 / 2)}}{E h^{5}} E_{i j} \text {, } \\
& \bar{F}_{i j}=\frac{(a b)^{(1 / 2)}}{E h^{6}} F_{i j} \text {, } \\
& \bar{H}_{i j}=\frac{(a b)^{(1 / 2)}}{E h^{8}} H_{i j} \text {, } \\
& \bar{I}_{i}=\frac{1}{(a b)^{(i+1 / 2)} \rho} I_{i}, \\
& \bar{\gamma}=\frac{(a b)^{2}}{\pi^{2} h^{4}(\rho E)^{(1 / 2)}} \gamma, \\
& \bar{x}=\frac{x}{a}, \\
& \bar{y}=\frac{b}{y}, \\
& \bar{\Omega}_{i}=\frac{1}{\pi^{2}}\left(\frac{E}{a b \rho}\right)^{(1 / 2)} \Omega_{i}, \\
& \bar{F}=\frac{b^{2}}{E h^{3}} F .
\end{aligned}
$$

\section{Frequency Analysis}

High-dimensional nonlinear dynamic systems contain several types of the internal resonant cases which can lead to different forms of the nonlinear vibrations. When the system exists a special internal resonant relationship between two linear modes, the large amplitude nonlinear responses may 
suddenly happen which is dangerous in engineering and should be avoided.

In order to study the internal resonance relationship between two bending modes, the finite element model of the cantilever laminated composite plate is established. The ply stacking sequence is $\left[0^{\circ} / 90^{\circ}\right]_{S}$ and the material parameters [51] are shown in Table 2.

The natural frequencies of bending vibration of laminated composite cantilever plates with different span-chord ratio and different layer thickness are calculated, and the results are shown in Figures 5-8. When the span-chord ratio is $2: 1$ and single thickness is $6 \mathrm{~mm}$, the transverse vibration modes of the cantilever laminated composite plate with corresponding frequencies are shown in Figure 9.

In frequency analysis, the first mode natural frequency is most important. Table 3 is to illustrate the 1st mode natural frequency for 6 (thickness values) $\times 4$ (span-chord ratio values) from Figures 5-8.

Based on the results of numerical simulations, the first six orders natural frequencies of the laminated composite cantilever plate are obtained, as shown in Figures $5-8$. It is obviously observed that there is a proportional relation between the two bending modes, such as relation $1: 1$ in area $c$ of Figure 7, relation $1: 2$ in Figure 8, and relation $1: 3$ in Figures $5-7$. We select $1: 2$ internal resonance relationship between two bending modes and the nonlinear vibrations of the laminated composite cantilever plate are investigated in the following analysis.

\section{Perturbation Analysis}

The discrete equation is derived by the Galerkin method, and the discrete function adopts the following expression:

$$
w_{0}=w(t)_{1} X_{1}(x) Y_{1}(y)+w(t)_{2} X_{2}(x) Y_{2}(y)
$$

where

$$
X_{i}(x)=\sin \frac{\lambda_{i}}{a} x-\sinh \frac{\lambda_{i}}{a} x+\alpha_{i}\left(\cosh \frac{\lambda_{i}}{a} x-\cos \frac{\lambda_{i}}{a} x\right),
$$

$Y_{j}(y)=\sin \frac{\beta_{m}}{b} y+\sinh \frac{\beta_{m}}{b} y-\alpha_{m}\left(\cosh \frac{\beta_{m}}{b} y+\cos \frac{\beta_{m}}{b} y\right)$,

where

$$
\begin{gathered}
\cos \lambda_{i} a \cosh \lambda_{i} a-1=0, \\
\cos \beta_{m} b \cosh \beta_{m} b-1=0, \\
\alpha_{i}=\frac{\cosh \lambda_{i}-\cos \lambda_{i}}{\sinh \lambda_{i}+\sin \lambda_{i}}, \\
\alpha_{m}=-\frac{\cosh \beta_{m}-\cos \beta_{m}}{\sinh \beta_{m}-\sin \beta_{m}} .
\end{gathered}
$$

TABLE 2: Single-layer material setting.

\begin{tabular}{lcccc}
\hline Material & $E_{11}$ & $E_{22}$ & $S_{12}$ & $v$ \\
\hline T300/QY8911 & $135 \mathrm{GPa}$ & $88 \mathrm{GPa}$ & $45 \mathrm{GPa}$ & 0.3 \\
\hline
\end{tabular}

The single layer thickness is $h_{i}(i=1,2, \ldots, 6)$ and they are specified as $h_{1}=1 \mathrm{~mm}, h_{2}=2 \mathrm{~mm}, \ldots, h_{6}=6 \mathrm{~mm}$.

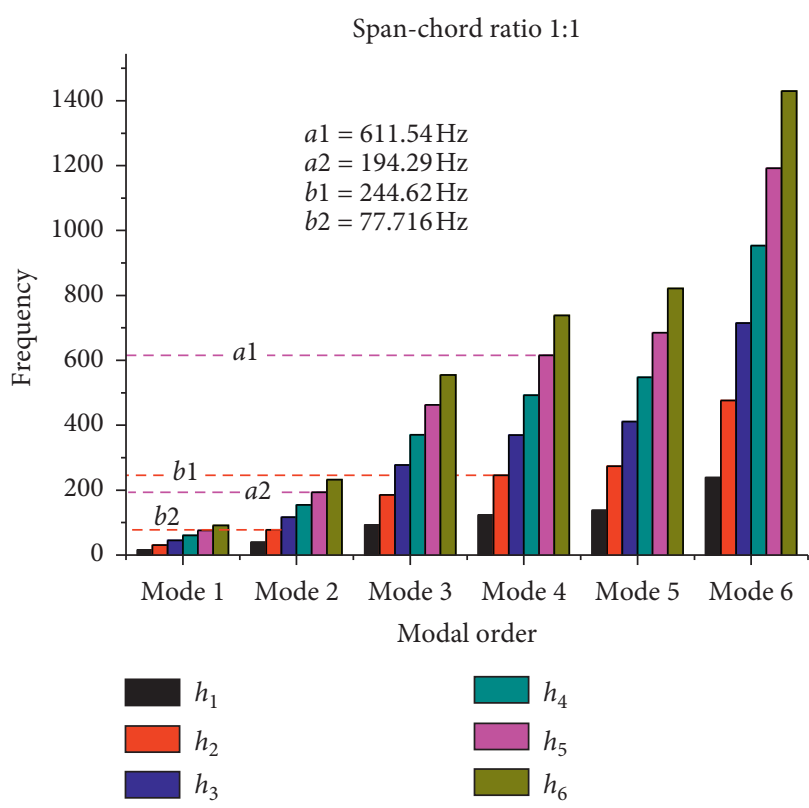

Figure 5: Natural frequencies with different thicknesses under the span-chord ratio $1: 1$.

Similarly, the aerodynamic force is discretized by using the modal function:

$$
L=l_{1} X_{1}(x) Y_{1}(y)+l_{2} X_{2}(x) Y_{2}(y),
$$

where $l_{1}$ and $l_{2}$ represent the amplitudes of the aerodynamic forces corresponding to the two transvers vibration modes and they contain the perturbed items.

Substituting equations (30)-(33) into equation (A.3), the governing differential equations of transverse motion of the system are derived as follows:

$$
\begin{aligned}
& \ddot{w}_{1}+\gamma_{11} \dot{w}_{1}+\omega_{1}^{2} w_{1}+\gamma_{12} f_{1} \cos \Omega_{1} t w_{1}+\gamma_{13} w_{2}-\gamma_{14} w_{1}^{3} \\
& \quad-\gamma_{15} w_{1}^{2} w_{2}-\gamma_{16} w_{1} w_{2}^{2}-\gamma_{17} w_{2}^{3}=\gamma_{18} l_{1}, \\
& \ddot{w}_{2}+\gamma_{21} \dot{w}_{2}+\omega_{2}^{2} w_{2}+\gamma_{22} f_{1} \cos \Omega_{1} t w_{2}+\gamma_{23} w_{1}-\gamma_{24} w_{2}^{3} \\
& \quad-\gamma_{25} w_{2}^{2} w_{1}-\gamma_{26} w_{2} w_{1}^{2}-\gamma_{27} w_{1}^{3}=\gamma_{28} l_{2} .
\end{aligned}
$$




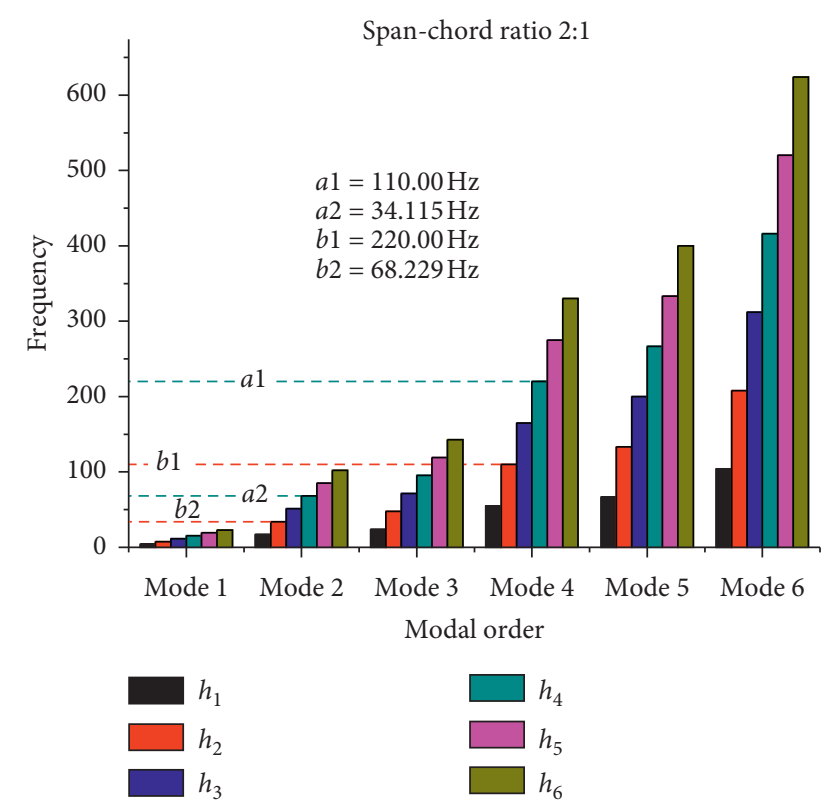

FIGURE 6: Natural frequencies with different thicknesses under the span-chord ratio $2: 1$.

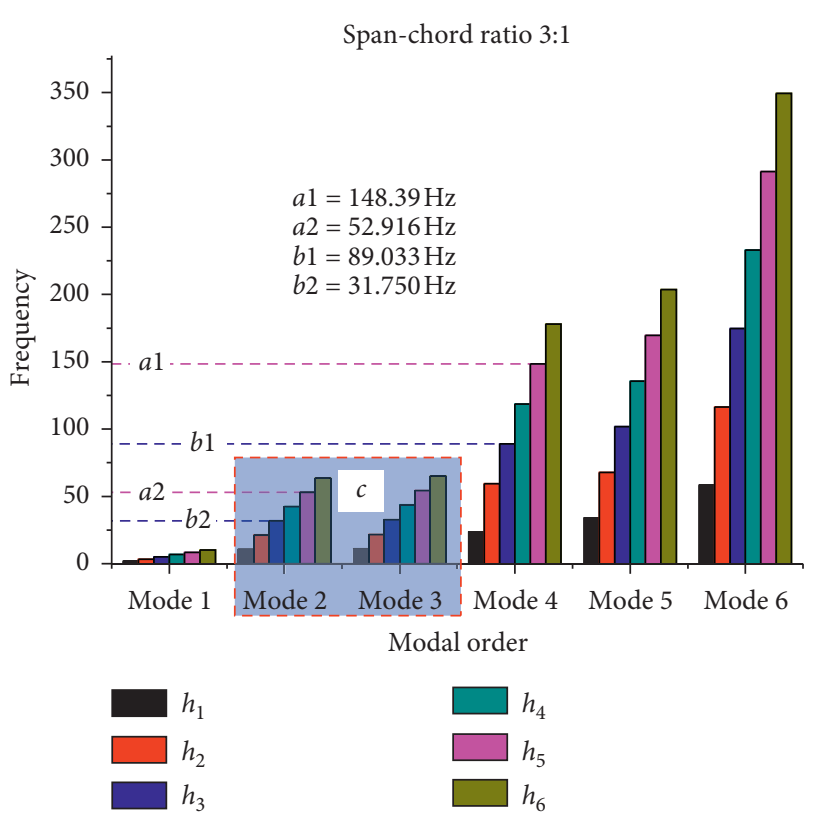

FIGURE 7: Natural frequencies with different thicknesses under the span-chord ratio $3: 1$.

Next, the averaged equations of the system are obtained by using the multiple scale method, and the internal resonant relationship $1: 2$ is considered:

$$
\begin{aligned}
& \omega_{1}^{2}=\frac{1}{4} \Omega^{2}+\varepsilon \sigma_{1}, \\
& \omega_{2}^{2}=\Omega^{2}+\varepsilon \sigma_{2}, \\
& \Omega_{1}=\Omega_{2}=1,
\end{aligned}
$$

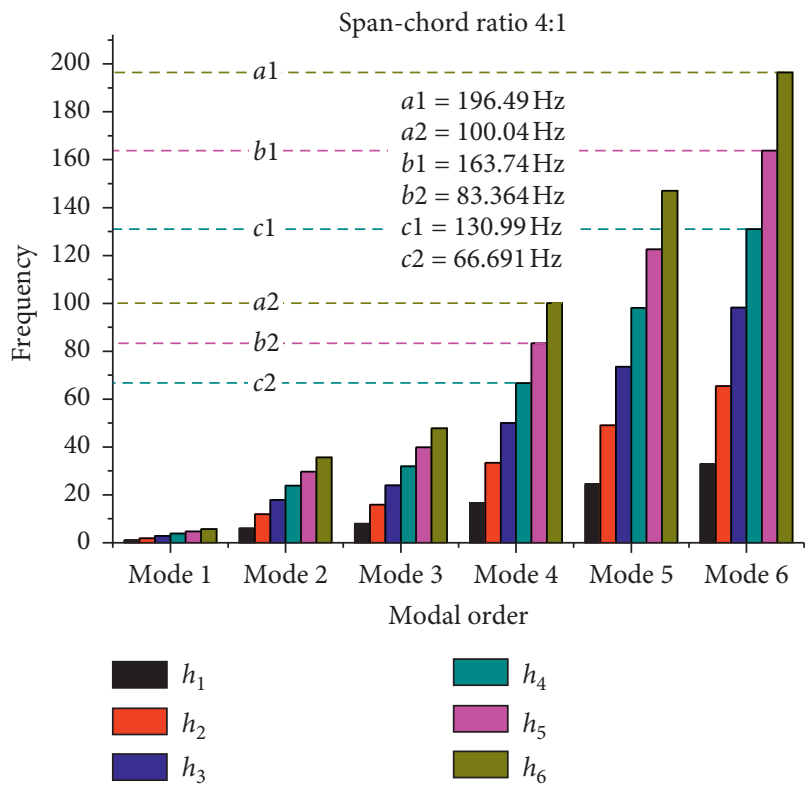

Figure 8: Natural frequencies with different thicknesses under the span-chord ratio $4: 1$.

where $\omega_{1}$ and $\omega_{2}$ are two frequencies of the laminated composite plate and $\sigma_{1}$ and $\sigma_{2}$ are the tuning parameters.

The scale transformations are given as follows:

$$
\begin{aligned}
& \gamma_{11} \longrightarrow \varepsilon \gamma_{11}, \\
& \gamma_{12} \longrightarrow \varepsilon \gamma_{12}, \\
& \gamma_{13} \longrightarrow \varepsilon \gamma_{13}, \\
& \gamma_{14} \longrightarrow \varepsilon \gamma_{14}, \\
& \gamma_{15} \longrightarrow \varepsilon \gamma_{15}, \\
& \gamma_{16} \longrightarrow \varepsilon \gamma_{16}, \\
& \gamma_{17} \longrightarrow \varepsilon \gamma_{17}, \\
& \gamma_{18} \longrightarrow \varepsilon \gamma_{18}, \\
& \gamma_{21} \longrightarrow \varepsilon \gamma_{21}, \\
& \gamma_{22} \longrightarrow \varepsilon \gamma_{22}, \\
& \gamma_{23} \longrightarrow \varepsilon \gamma_{23}, \\
& \gamma_{24} \longrightarrow \varepsilon \gamma_{24}, \\
& \gamma_{25} \longrightarrow \varepsilon \gamma_{25}, \\
& \gamma_{26} \longrightarrow \varepsilon \gamma_{26}, \\
& \gamma_{27} \longrightarrow \varepsilon \gamma_{27}, \\
& \gamma_{28} \longrightarrow \varepsilon \gamma_{28} .
\end{aligned}
$$

The scale transformation can be obtained by introducing equation (36) into equations (34a) and (34b):

$$
\begin{aligned}
& \ddot{w}_{1}+\varepsilon \gamma_{11} \dot{w}_{1}+\omega_{1}^{2} w_{1}+\varepsilon \gamma_{12} f_{1} \cos \Omega_{1} t w_{1}+\varepsilon \gamma_{13} w_{2} \\
& \quad-\varepsilon \gamma_{14} w_{1}^{3}-\varepsilon \gamma_{15} w_{1}^{2} w_{2}-\varepsilon \gamma_{16} w_{1} w_{2}^{2}-\varepsilon \gamma_{17} w_{2}^{3}=\varepsilon \gamma_{18} l_{1}, \\
& \ddot{w}_{2}+\varepsilon \gamma_{21} \dot{w}_{2}+\omega_{2}^{2} w_{2}+\varepsilon \gamma_{22} f_{1} \cos \Omega_{1} t w_{2}+\varepsilon \gamma_{23} w_{1} \\
& \quad-\varepsilon \gamma_{24} w_{2}^{3}-\varepsilon \gamma_{25} w_{2}^{2} w_{1}-\varepsilon \gamma_{26} w_{2} w_{1}^{2}-\varepsilon \gamma_{27} w_{1}^{3}=\varepsilon \gamma_{28} l_{2}
\end{aligned}
$$




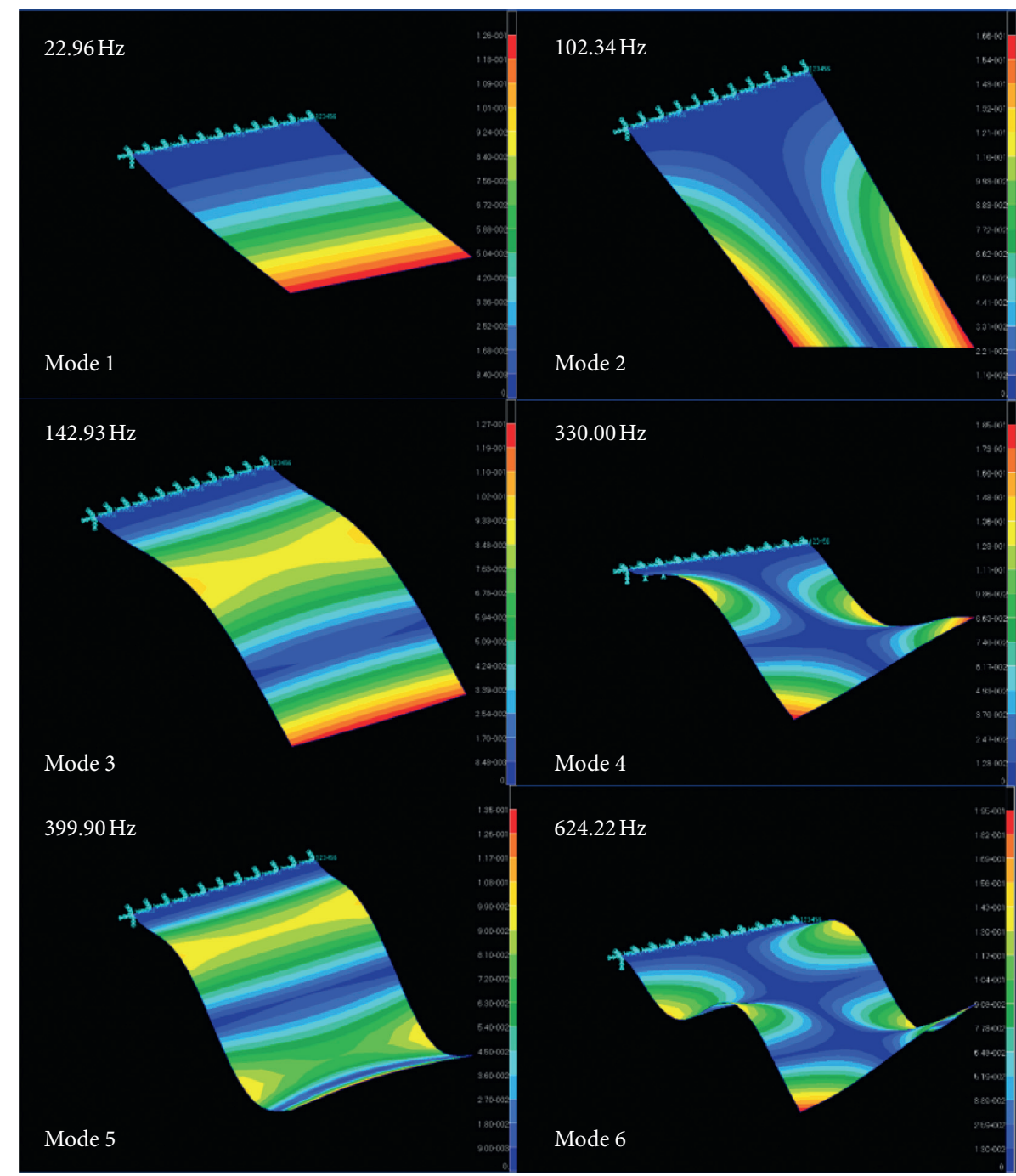

FIGURE 9: The transverse modes of the cantilever laminated composite plate when the span-chord ratio is $2: 1$ and single thickness is 6 mm.

TABLE 3: The 1st mode natural frequency for four span-chord ratio values corresponding to the thickness.

\begin{tabular}{lcccccc}
\hline \multirow{2}{*}{ Span-chord ratio } & \multicolumn{7}{c}{ Frequency $(\mathrm{Hz})$} \\
& $h_{1}$ & $h_{2}$ & $h_{3}$ & $h_{4}$ & $h_{5}$ & $h_{6}$ \\
\hline $1: 1$ & 15.15 & 30.31 & 45.46 & 60.61 & 75.77 & 90.92 \\
$2: 1$ & 3.83 & 7.65 & 11.48 & 15.31 & 19.13 & 22.96 \\
$3: 1$ & 1.69 & 3.39 & 5.08 & 6.78 & 8.47 & 10.17 \\
$4: 1$ & 0.95 & 1.90 & 2.85 & 3.80 & 4.75 & 5.70 \\
\hline
\end{tabular}

The method of multiple scales [52] is used to obtain the averaged equations in following form:

$$
w(x, t, \varepsilon)=w_{0}\left(x, T_{0}, T_{1}\right)+\varepsilon w_{1}\left(x, T_{0}, T_{1}\right),
$$

where $T_{0}=t$ and $T_{1}=\varepsilon t$.

The derivatives with respect to $t$ become

$$
\begin{gathered}
\frac{d}{d t}=\frac{\partial}{\partial T_{0}} \frac{d T_{0}}{d t}+\frac{\partial}{\partial T_{1}} \frac{d T_{1}}{d t}+\cdots=D_{0}+\varepsilon D_{1}+\cdots, \\
\frac{d^{2}}{d t^{2}}=\left(D_{0}+\varepsilon D_{1}+\cdots\right)^{2}=D_{0}^{2}+2 \varepsilon D_{0} D_{1}+\cdots,
\end{gathered}
$$

where $D_{n}=\left(\partial / \partial T_{n}\right), n=0,1,2, \ldots$..

Substituting equations (38)-(39b) into equations (37a) and (37b) and eliminating the secular terms, we have the averaged equations as follows:

$$
\begin{aligned}
D_{1} A_{1}= & -\frac{1}{2} \gamma_{11} A_{1}+i \sigma_{1} A_{1}+\frac{1}{2} i \gamma_{12} f_{1} \bar{A}_{1}-3 i A_{1}^{2} \bar{A}_{1} \gamma_{13} \\
& -2 i \gamma_{16} A_{1} A_{2} \bar{A}_{2}, \\
D_{1} A_{2}= & -\frac{1}{2} \gamma_{21} A_{2}+\frac{1}{2} i \sigma_{2} A_{2}-\frac{3}{2} i \gamma_{23} A_{2}^{2} \bar{A}_{2}-i \gamma_{26} A_{1} A_{2} \bar{A}_{1} \\
& -\frac{1}{4} i \gamma_{28} L_{2} .
\end{aligned}
$$

In order to obtain the averaged equations in the polar coordinate system, we express $A_{1}$ and $A_{2}$ in the following form: 


$$
\begin{aligned}
& A_{1}\left(T_{1}\right)=\frac{1}{2} a_{1}\left(T_{1}\right) e^{j \phi\left(T_{1}\right)}, \\
& A_{2}\left(T_{2}\right)=\frac{1}{2} a_{2}\left(T_{2}\right) e^{j \phi\left(T_{2}\right)} .
\end{aligned}
$$

Substituting equation (41) into equations (40a) and (40b) and separating the real and imaginary parts, the four-dimensional averaged equations in the polar coordinate system are obtained:

$$
\begin{aligned}
\dot{a}_{1} & =-\frac{1}{4}\left(\gamma_{11} a_{1} \sin \phi_{1}-2 \sigma_{1} a_{1} \cos \phi_{1}-\gamma_{12} a_{1} f_{1} \cos \phi_{1}+\frac{3}{2} \gamma_{14} a_{1}^{3} \cos \phi_{1}+\gamma_{16} a_{1} a_{2}^{2} \sin \phi_{1}\right), \\
a_{1} \dot{\phi}_{1} & =-\frac{1}{4}\left(\gamma_{11} a_{1} \cos \phi_{1}+2 \sigma_{1} a_{1} \sin \phi_{1}+\gamma_{12} a_{1} f_{1} \sin \phi_{1}-\frac{3}{2} \gamma_{14} a_{1}^{3} \sin \phi_{1}+\gamma_{16} a_{1} a_{2}^{2} \cos \phi_{1}\right), \\
\dot{a}_{2} & =-\frac{1}{4}\left(\gamma_{21} a_{2} \sin \phi_{2}-\sigma_{2} a_{2} \cos \phi_{2}+\frac{3}{4} \gamma_{24} a_{2}^{3} \cos \phi_{2}+\frac{1}{2} \gamma_{26} a_{2} a_{1}^{2} \sin \phi_{2}+\gamma_{28} l_{2}\right), \\
a_{2} \dot{\phi}_{2} & =-\frac{1}{4}\left(\gamma_{21} a_{2} \cos \phi_{2}+\sigma_{2} a_{2} \sin \phi_{2}+\frac{3}{4} \gamma_{24} a_{2}^{3} \sin \phi_{2}+\frac{1}{2} \gamma_{26} a_{2} a_{1}^{2} \cos \phi_{2}\right) .
\end{aligned}
$$

In order to obtain the averaged equations in the Cartesian coordinate system, we rewrite $A_{1}$ and $A_{2}$ in the following forms:

$$
\begin{aligned}
& A_{1}\left(T_{1}\right)=x_{1}\left(T_{1}\right)+i x_{2}\left(T_{1}\right), \\
& A_{2}\left(T_{1}\right)=x_{3}\left(T_{1}\right)+i x_{4}\left(T_{1}\right) .
\end{aligned}
$$

According to the same way as the above, the averaged equations in the Cartesian coordinate system are obtained as follows:

$$
\begin{aligned}
\dot{x}_{1}= & -\frac{1}{2} \gamma_{11} x_{1}-\left(\sigma_{1}-\frac{1}{2} \gamma_{12} f_{1}\right) x_{2}+3 \gamma_{14} x_{2}\left(x_{1}^{2}+x_{2}^{2}\right) \\
& +2 \gamma_{16} x_{2}\left(x_{3}^{2}+x_{4}^{2}\right), \\
\dot{x}_{2}= & -\frac{1}{2} \gamma_{11} x_{2}+\left(\sigma_{1}+\frac{1}{2} \gamma_{12} f_{1}\right) x_{1}-3 \gamma_{14} x_{1}\left(x_{1}^{2}+x_{2}^{2}\right) \\
& -2 \gamma_{16} x_{1}\left(x_{3}^{2}+x_{4}^{2}\right), \\
\dot{x}_{3}= & -\frac{1}{2} \gamma_{21} x_{3}-\frac{1}{2} \sigma_{2} x_{4}+\frac{3}{2} \gamma_{24} x_{4}\left(x_{3}^{2}+x_{4}^{2}\right)+\gamma_{26} x_{4}\left(x_{1}^{2}+x_{2}^{2}\right), \\
\dot{x}_{4}= & -\frac{1}{2} \gamma_{21} x_{4}+\frac{1}{2} \sigma_{2} x_{3}-\frac{3}{2} \gamma_{24} x_{3}\left(x_{3}^{2}+x_{4}^{2}\right)-\gamma_{26} x_{3}\left(x_{1}^{2}+x_{2}^{2}\right) \\
& -\frac{1}{4} \gamma_{28} l_{2} .
\end{aligned}
$$

\section{Numerical Simulation}

In order to study the nonlinear vibration characteristics of the laminated composite cantilever plate with different modal modes, based on equations (44a) and (44d), the frequency-response curves are used to reveal the characteristics of this system. Let $\dot{a}_{1}=\dot{a}_{2}=\dot{\phi}_{1}=\dot{\phi}_{2}=0$, $\phi_{1}=(\pi / 4)$, and $\phi_{2}=(3 \pi / 4)$.

The frequency-response functions of the system are given as follows:

$$
\begin{aligned}
& 0=\gamma_{11} a_{1}-2 \sigma_{1} a_{1}-\gamma_{12} a_{1} f_{1}+\frac{3}{2} \gamma_{14} a_{1}^{3}+\gamma_{16} a_{1} a_{2}^{2}, \\
& 0=\gamma_{21} a_{2}+\sigma_{2} a_{2}-\frac{3}{4} \gamma_{24} a_{2}^{3}+\frac{1}{2} \gamma_{26} a_{2} a_{1}^{2}+\sqrt{2} \gamma_{28} l_{2} .
\end{aligned}
$$

From equations (45a)-(45b), we can find that the amplitude $a_{1}$ and amplitude $a_{2}$ are coupled. The case of weak coupled form is considered here. Through introducing the proportional relation $\left(a_{1} / a_{2}\right)=\varepsilon$, the frequency-responses between the amplitudes and the tuning parameters are analyzed.

According to the geometries and the material properties of the nonlinear system, the basic parameters are chosen as $\gamma_{11}=0.2, \quad \gamma_{12}=-6, \quad \gamma_{14}=5, \gamma_{16}=-5, \gamma_{21}=0.6, \quad \gamma_{24}=9$, $\gamma_{26}=9$, and $\gamma_{28}=-2$. The relationship between the amplitude and the tuning parameter in different excitation conditions can be obtained.

Figure 10 gives the relationship between the amplitude $a_{1}$ and tuning parameter $\sigma_{1}$ in different internal force amplitudes $f_{1}$, and Figure 11 gives the relationship between the amplitude $a_{2}$ and the tuning parameter $\sigma_{2}$ in different aerodynamic amplitudes $l_{2}$.

The stiffness hardening phenomenon of the system can be seen in the relationship between the tuning parameters and the amplitude. With the increase of external excitation amplitude, the stiffness hardening phenomenon is gradually strengthened. The typical jump phenomenon of the nonlinear oscillations also happened in the system. The jump phenomenon appeared in the frequency-response curves at point $A^{*}$ and point $B^{*}$ with the increase of the tuning parameters in Figure 11. The frequency-response curves have the wider resonance interval and the larger oscillation amplitudes under the stronger external excitation amplitude $l_{2}$. 


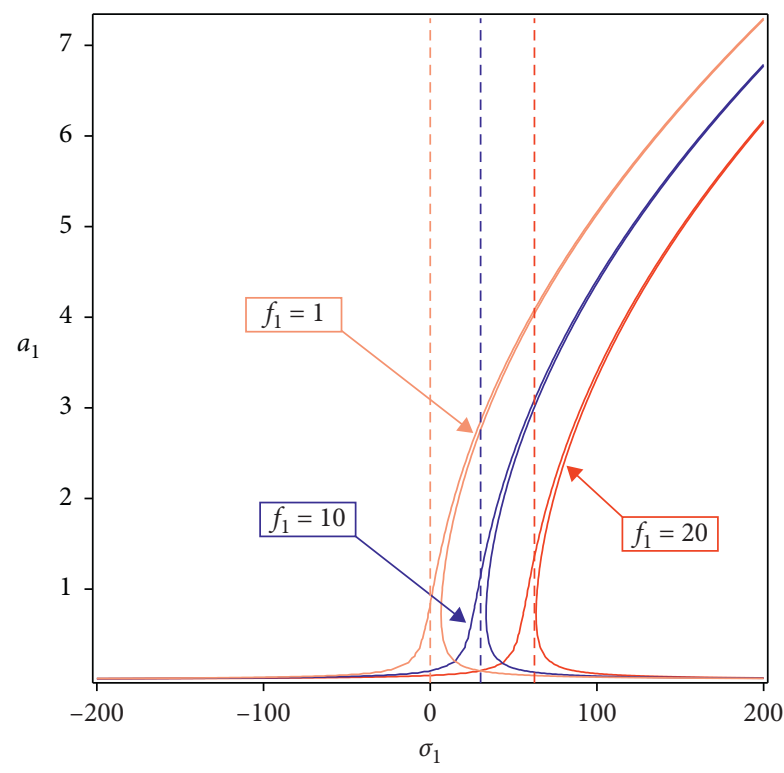

FIgURE 10: The relationship between amplitude $a_{1}$ and tuning parameter $\sigma_{1}$ in different internal force amplitudes $f_{1}$.

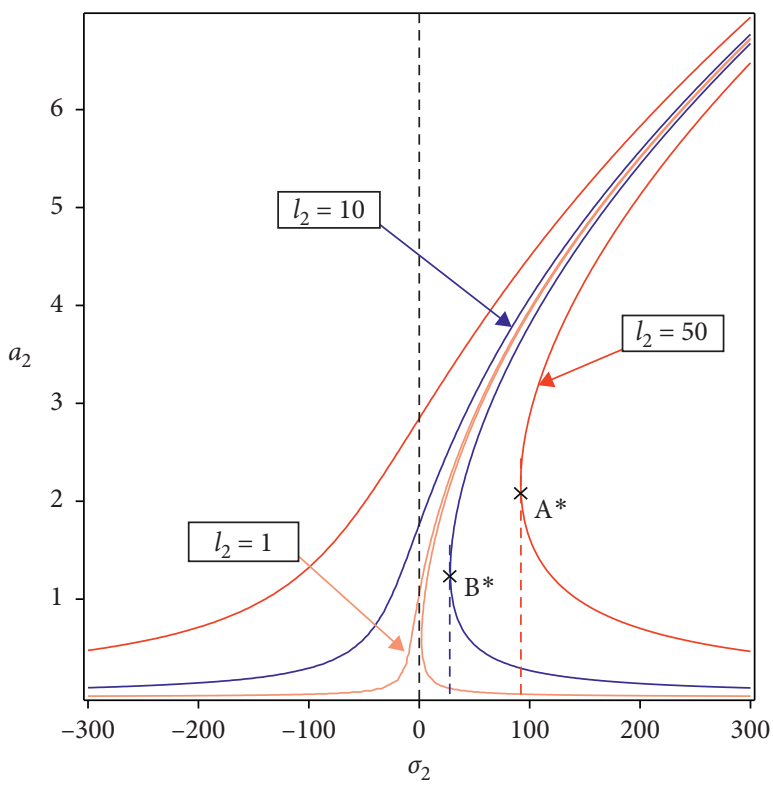

FIgURE 11: The relationship between amplitude $a_{2}$ and tuning parameter $\sigma_{2}$ in different aerodynamic amplitudes $l_{2}$.

Next, the geometries and the material properties of the nonlinear system are fixed, and the effects of different decoupling parameters $\varepsilon$ are calculated and the relationship between the amplitude and the tuning parameter can be found in Figures 12 and 13.

In the following investigation, the bifurcation and chaotic motions of the laminated composite cantilever plate based on the averaged equation in the case of one to two internal resonances by using the fourth-order Runge-Kutta algorithm are analyzed. We choose the force excitation $l_{2}$ as

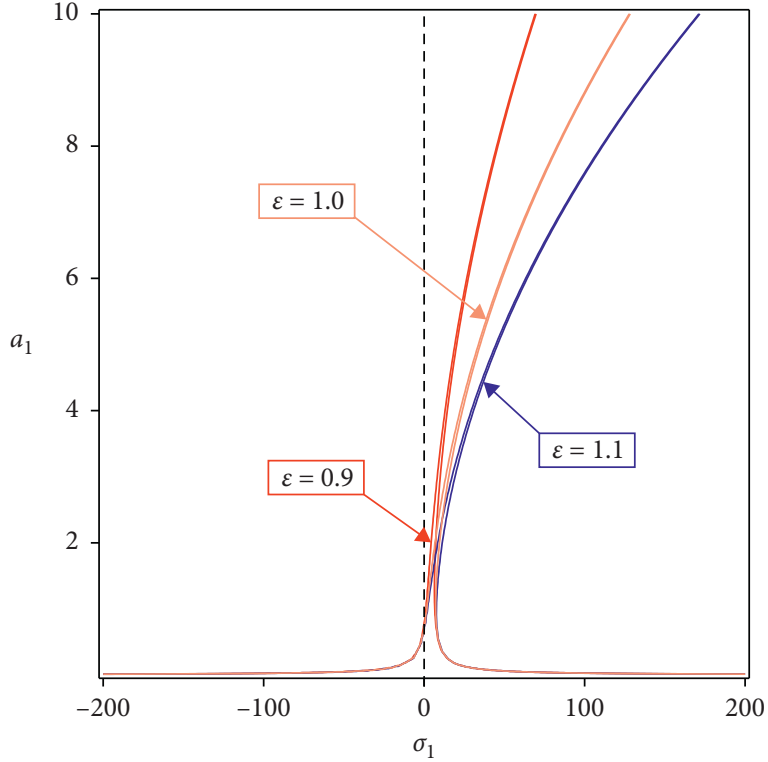

FIGURE 12: The relationship between amplitude $a_{1}$ and tuning parameter $\sigma_{1}$ in different decoupling parameters $\varepsilon$.

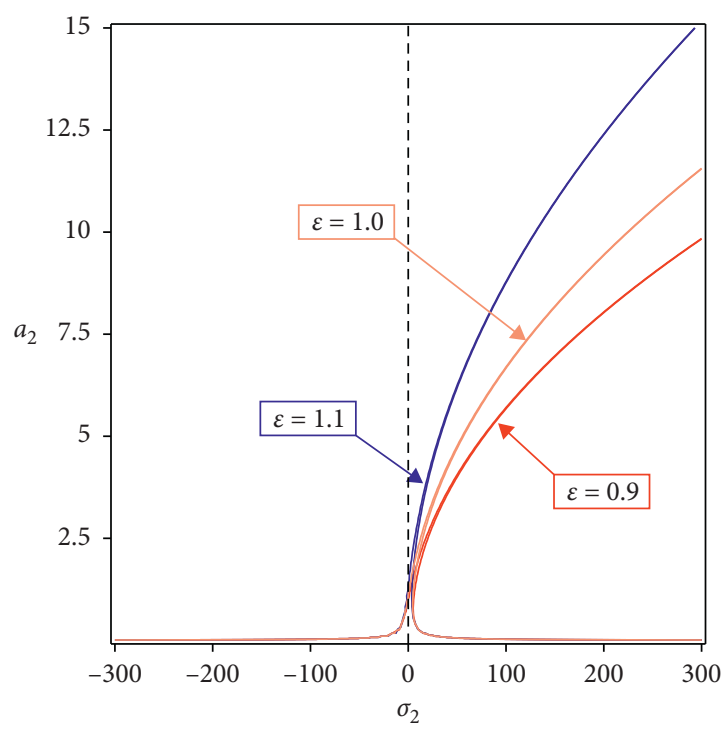

FIgURE 13: The relationship between amplitude $a_{2}$ and tuning parameter $\sigma_{2}$ in different decoupling parameters $\varepsilon$.

the controlling parameter to study the complicated nonlinear dynamics of the system.

The tuning parameters and the initial conditions are chosen as $\sigma_{1}=0.1, \sigma_{2}=1.1, \gamma_{11}=0.2, \gamma_{12}=5.2, \gamma_{14}=4.2$, $\gamma_{16}=-2.5, \gamma_{21}=0.529, \gamma_{24}=7, \gamma_{26}=5, \gamma_{28}=0.44, f_{1}=0.9$, $x_{10}=0.44, x_{20}=0.798, x_{30}=1.35$, and $x_{40}=-0.5$.

Figure 14 presents the two-dimensional bifurcation diagram to show the nonlinear oscillations of the laminated composite plate by varying the force excitation $l_{2}$. The periodic and chaotic motions of the laminated composite cantilever plate system for $x_{3}$ alternately occur with the increase of the parameter $l_{2}$ in the interval of $[20,50]$. 


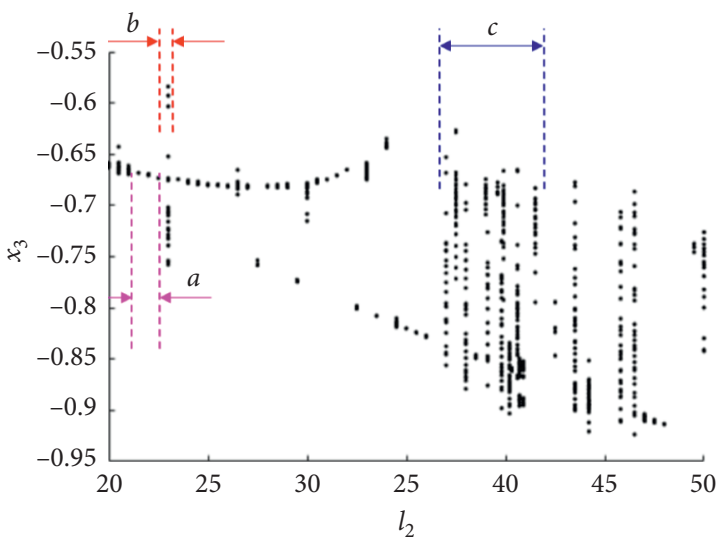

Figure 14: The bifurcation diagram of the laminated composite cantilever plate for $x_{3}$ via the forcing excitation $l_{2}$.

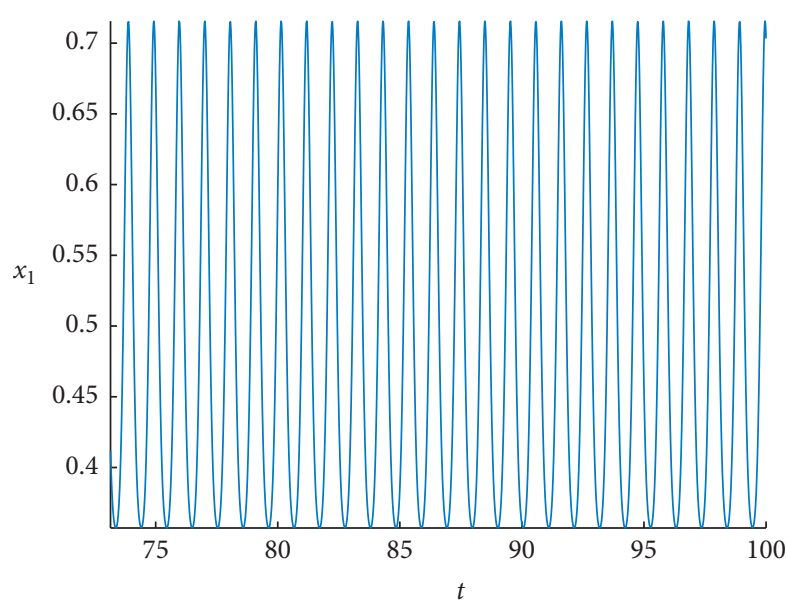

(a)

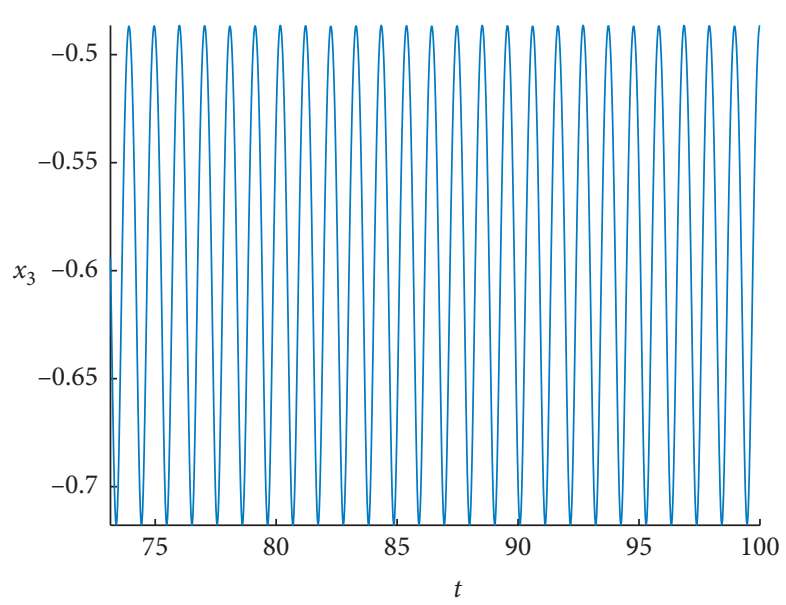

(c)

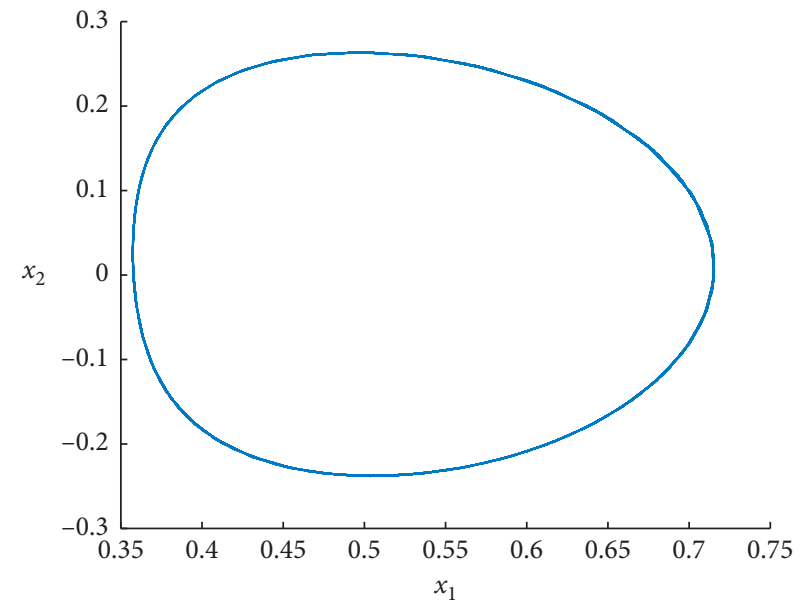

(b)

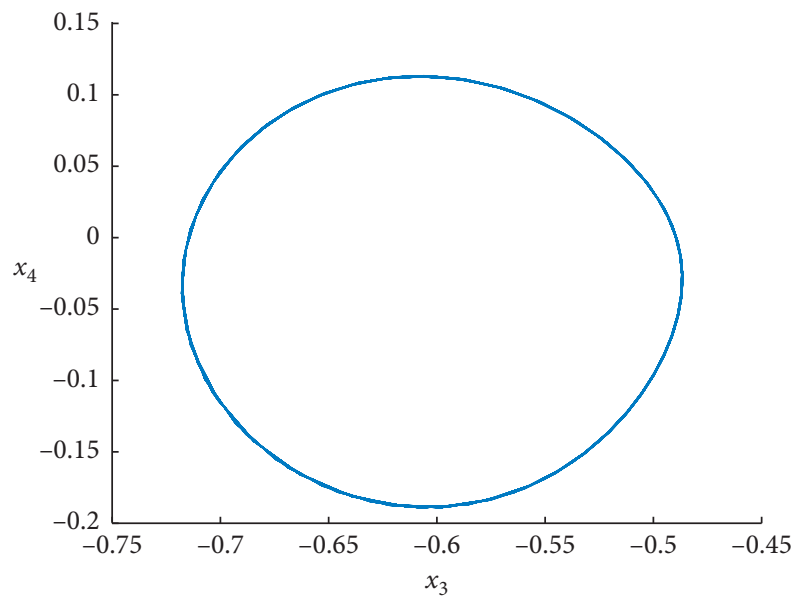

(d)

Figure 15: Continued. 


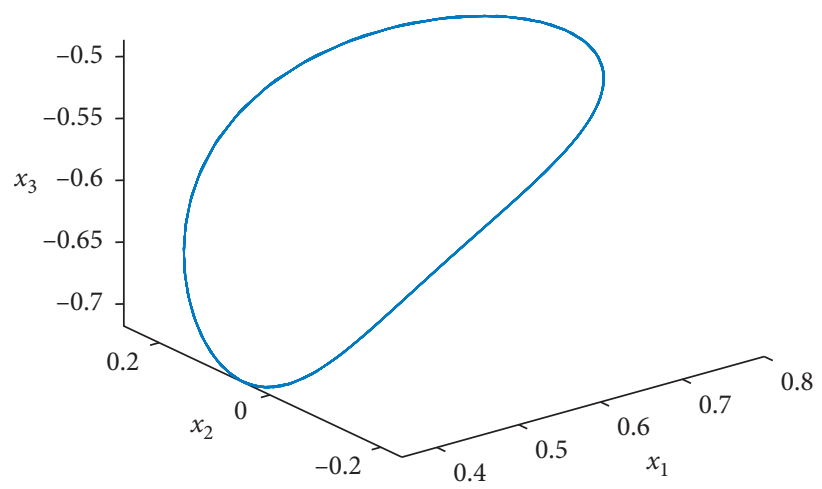

(e)

FIgURE 15: The period motion of the laminated composite cantilever plate is obtained when $l_{2}=21.5$.

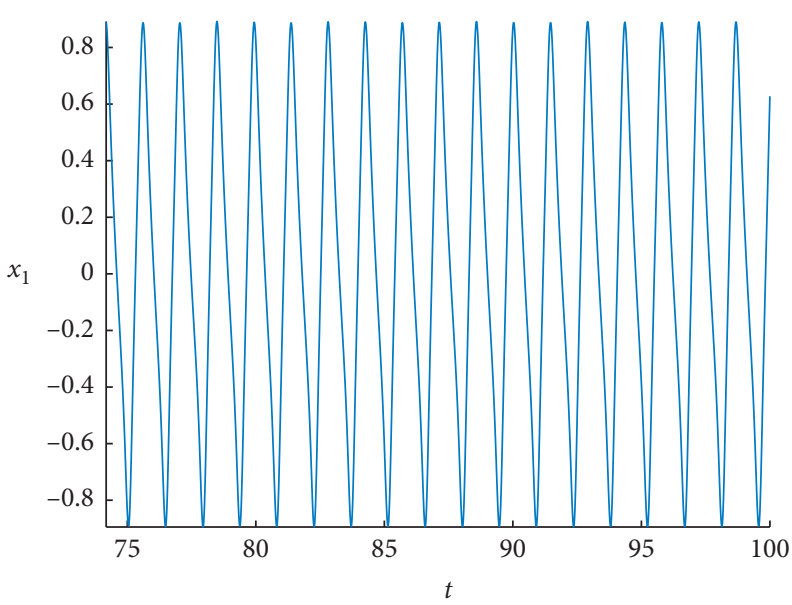

(a)

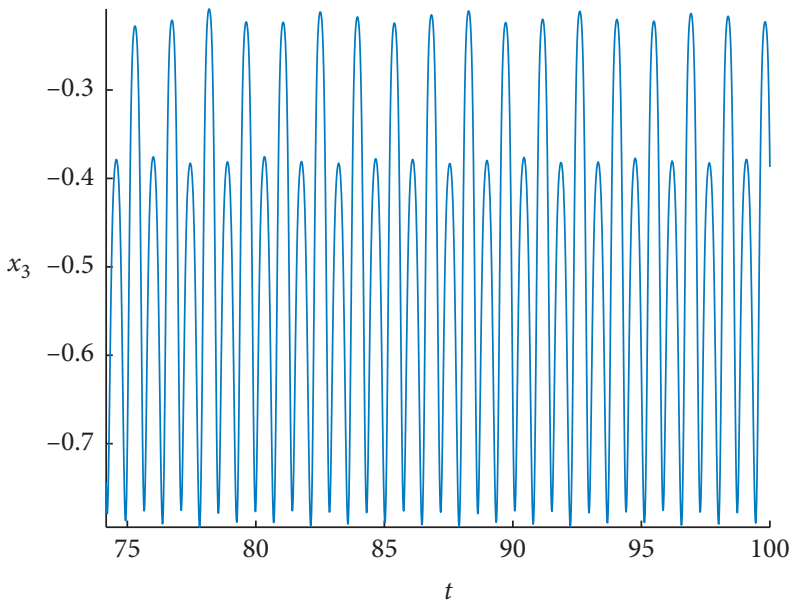

(c)

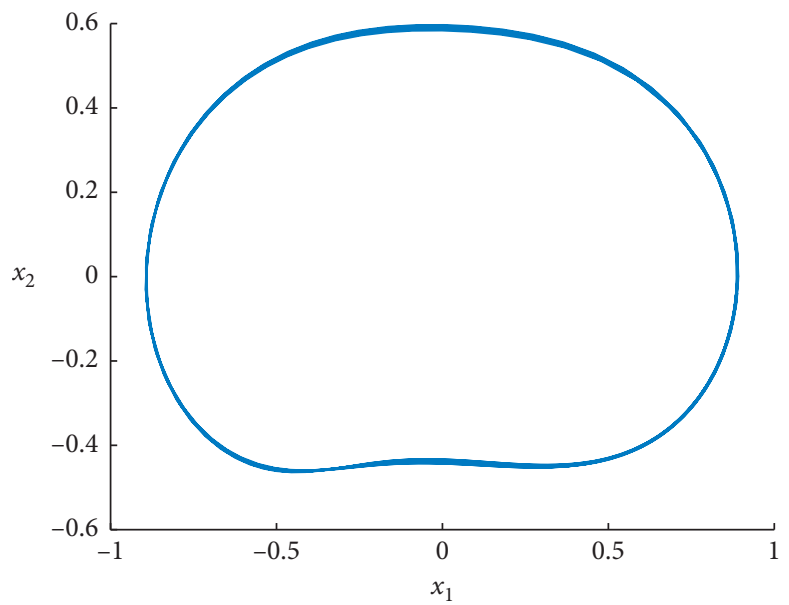

(b)

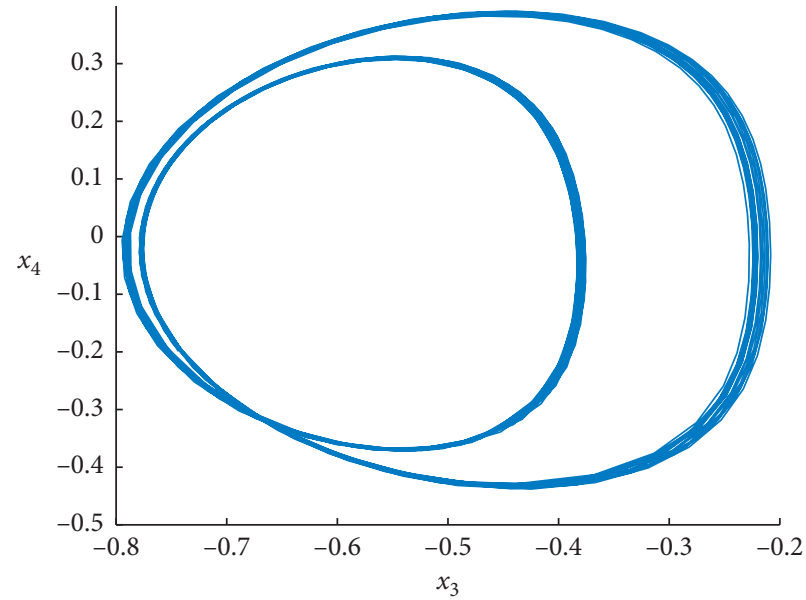

(d)

FIGURE 16: Continued. 


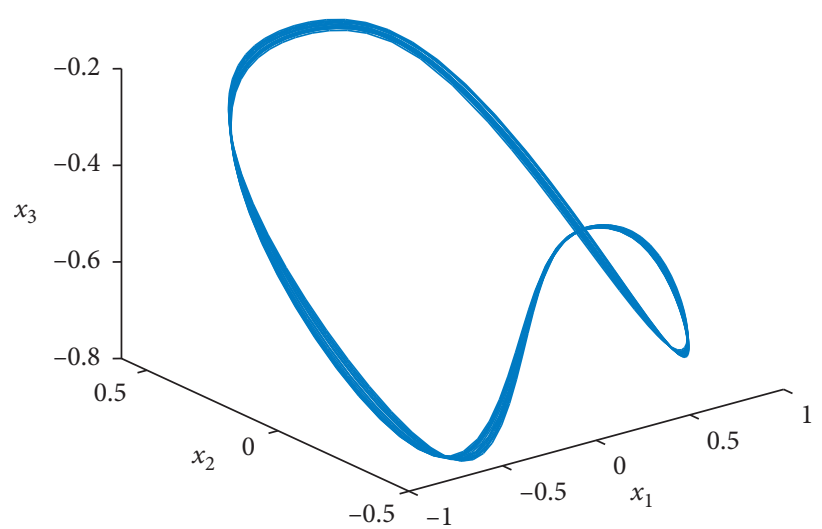

(e)

FIGURE 16: The multiperiod motion of the laminated composite cantilever plate is obtained when $l_{2}=22.7$.

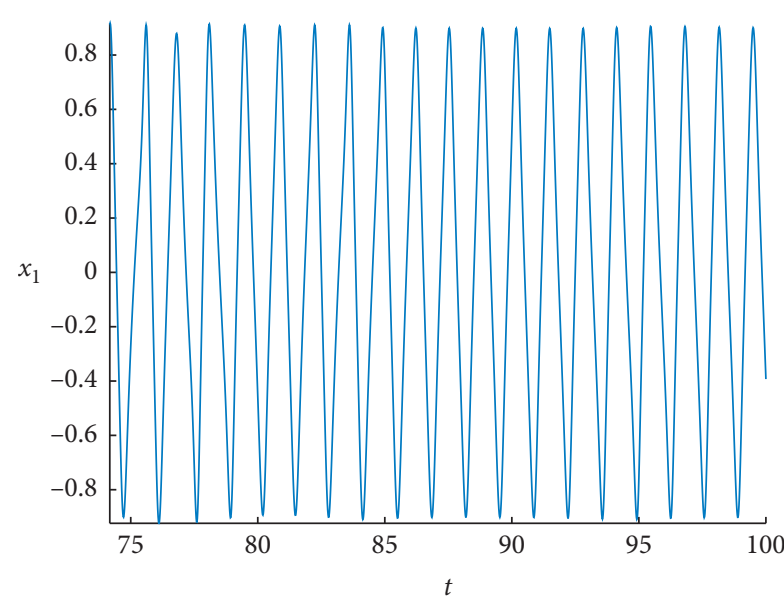

(a)

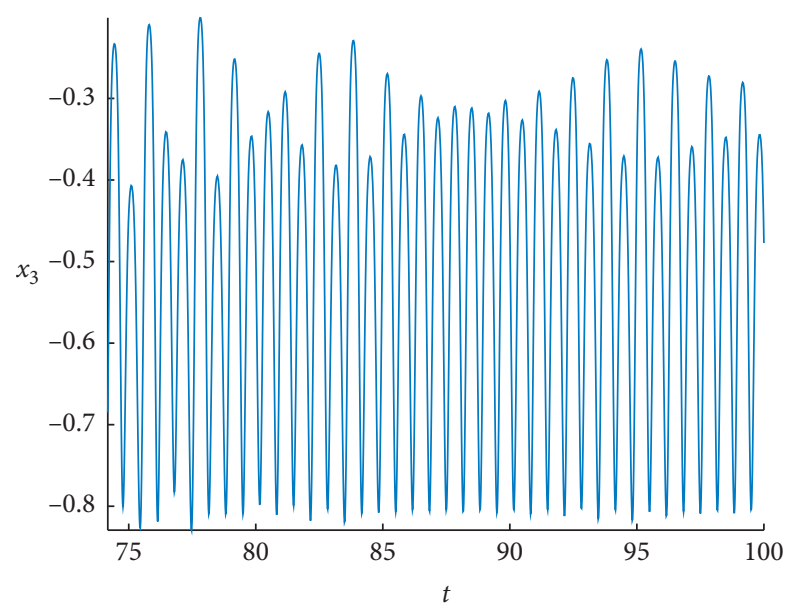

(c)

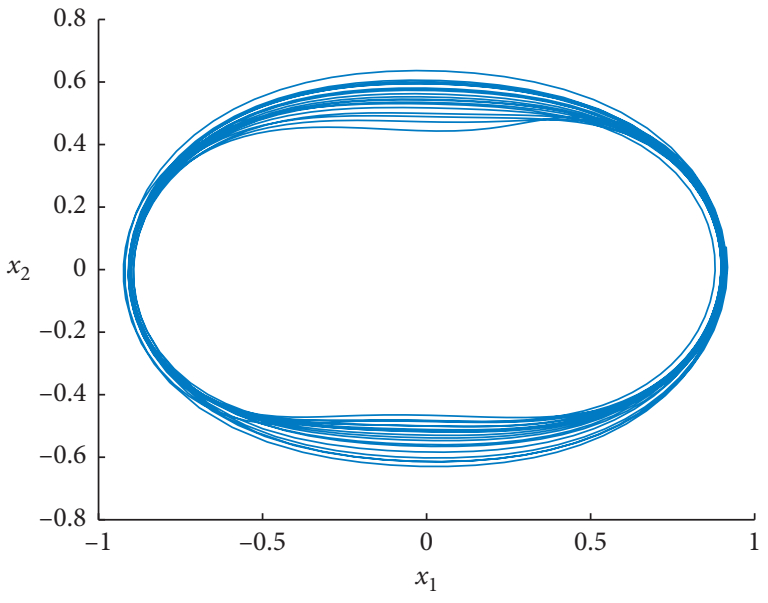

(b)

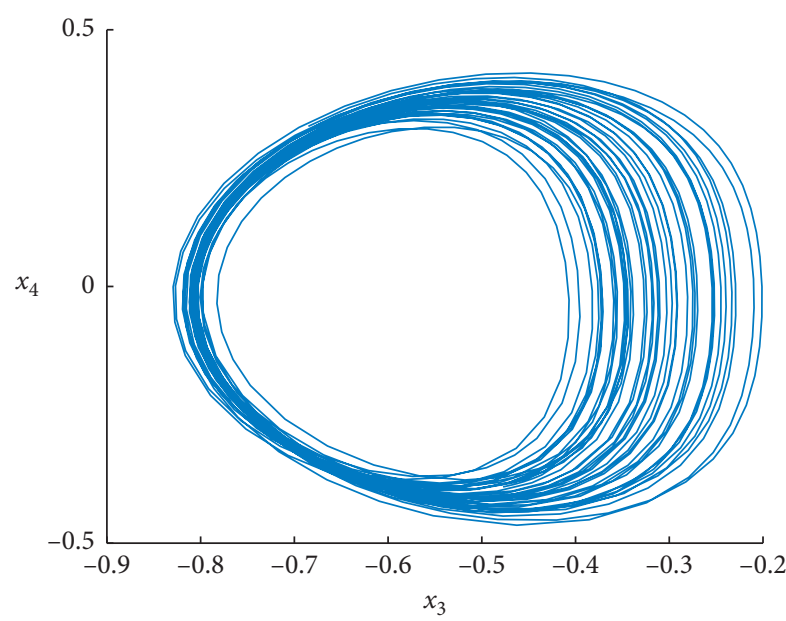

(d)

Figure 17: Continued. 


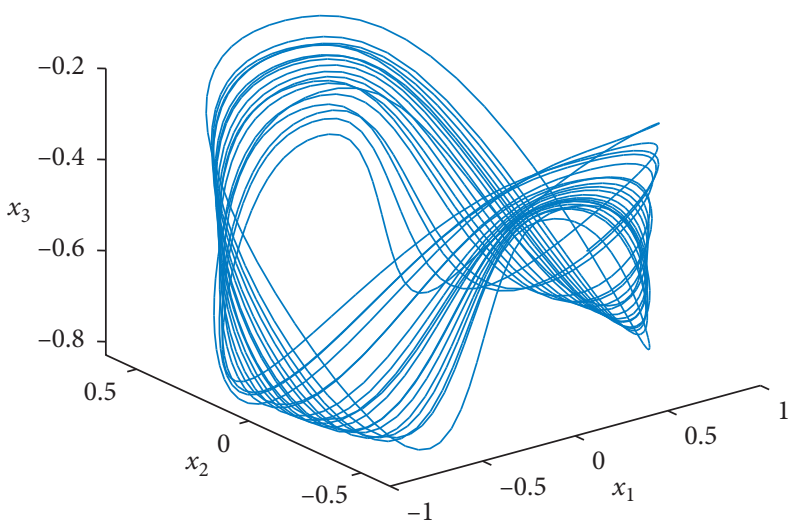

(e)

FIGURE 17: The quasi-period motion of the laminated composite cantilever plate is obtained when $l_{2}=25.3$.

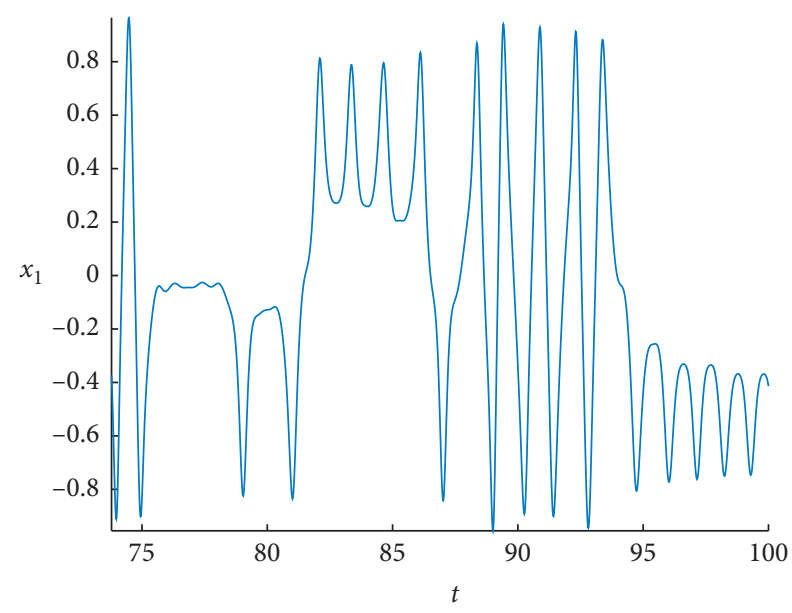

(a)

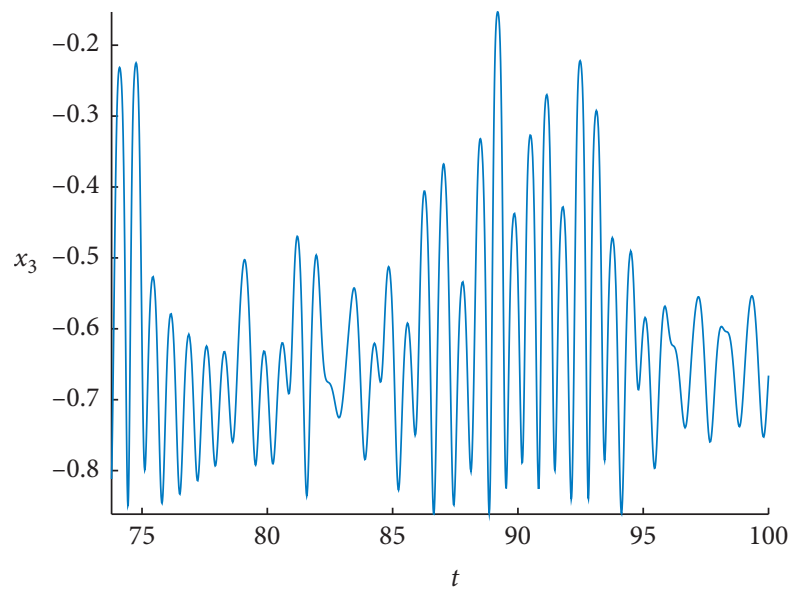

(c)

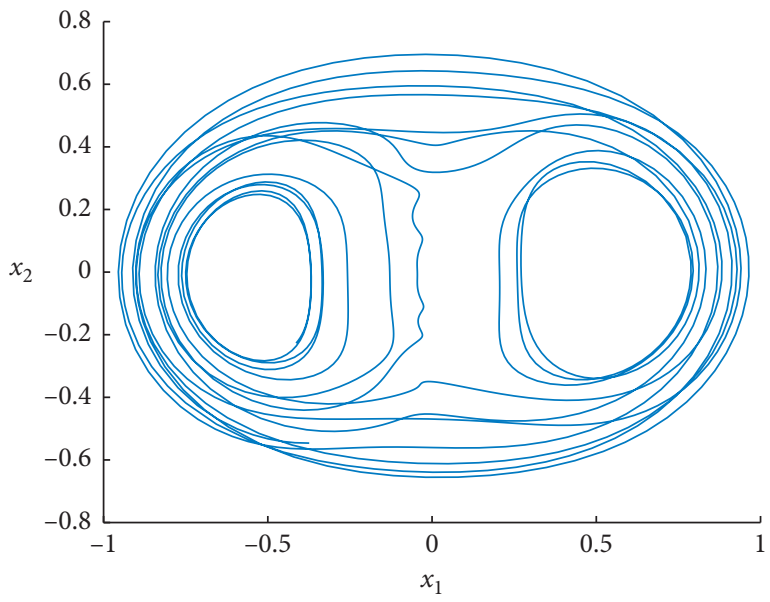

(b)

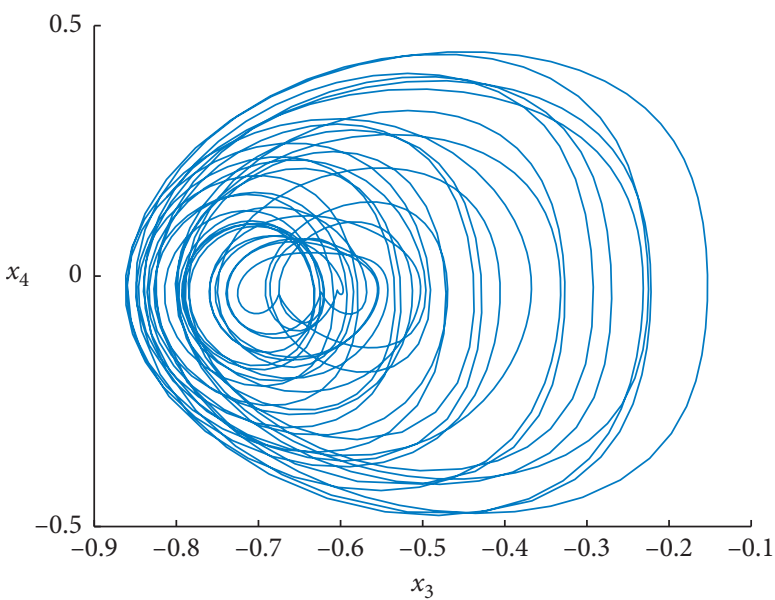

(d)

Figure 18: Continued. 


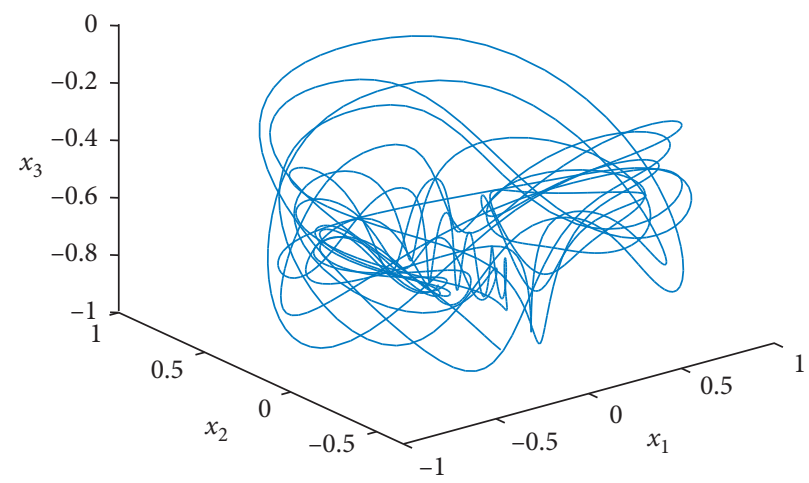

(e)

FIGURE 18: The chaotic motion of the laminated composite cantilever plate is obtained when $l_{2}=26.5$.

In Figures 15-18, (a) and (c) show the waveforms on the planes $\left(t, x_{1}\right)$ and $\left(t, x_{3}\right),(\mathrm{b})$ and $(\mathrm{d})$ depict the phase portraits on the planes $\left(x_{1}, x_{2}\right)$ and $\left(x_{3}, x_{4}\right)$, and (e) represents the three-dimensional phase portrait in the space $\left(x_{1}, x_{2}, x_{3}\right)$.

From Figures 15-18, the periodic and chaotic motions of the laminated composite cantilever plate occur with the increase of the amplitude of the parameter $l_{2}$ can be seen. It is noticed that both the power spectrum and the Poincaré map can distinguish the periodic motions and the chaotic motions. When the $l_{2}$ is in the range of $[21,27]$, the periodic motions and chaotic motions alternately occur, the system experiences periodic motion, multiperiodic motion, quasi-periodic motion, and chaotic motion. It is obvious that the nonlinear vibration characteristics of the system are complicated under the influence of external excitation.

\section{Conclusions}

The nonlinear dynamics of the laminated composite cantilever plate under subsonic excitation force have been investigated in this paper. The aerodynamic force of a threedimensional flat wing was calculated. Unlike a two-dimensional airfoil or an infinite length wing, the aerodynamic force of a three-dimensional flat wing is calculated. Some results are obtained:

(1) According to the ideal incompressible fluid flow condition and the Kutta-Joukowski lift theorem, the subsonic aerodynamic lift on the three-dimensional finite length flat wing is calculated by using the vortex lattice (VL) method.
(2) The finite length flat wing is modeled as a laminated composite cantilever plate based on Reddy's thirdorder shear deformation plate theory and the von Karman geometry nonlinearity is introduced. The nonlinear partial differential governing equations of motion for the laminated composite cantilever plate subjected to the subsonic aerodynamic force are established via Hamilton's principle.

(3) Through comparing the natural frequencies of the linear system with different materials and geometry parameters, the relationship of $1: 2$ internal resonance is found and considered for the nonlinear vibration analysis.

(4) Corresponding to several selected parameters, the frequency-response curves are obtained. The hardening-spring-type behaviors and jump phenomena are exhibited with the variation of the tuning parameters.

(5) The influence of the force excitation on the bifurcations and chaotic behaviors of the laminated composite cantilever plate have been investigated numerically. The periodic motion, multiperiodic motion, quasi-periodic motion, and chaotic motion of the system occurred with the increasing of the external excitation.

\section{Appendix}

$$
\begin{aligned}
& A_{11} \frac{\partial^{2} u_{0}}{\partial x^{2}}+A_{66} \frac{\partial^{2} u_{0}}{\partial y^{2}}+\left(A_{12}+A_{66}\right) \frac{\partial^{2} v_{0}}{\partial x \partial y}+A_{11} \frac{\partial w_{0}}{\partial x} \frac{\partial^{2} w_{0}}{\partial x^{2}}+A_{66} \frac{\partial w_{0}}{\partial x} \frac{\partial^{2} w_{0}}{\partial y^{2}}+\left(A_{12}+A_{66}\right) \frac{\partial w_{0}}{\partial y} \frac{\partial^{2} w_{0}}{\partial x \partial y}=I_{0} \ddot{u}_{0}+J \ddot{\varphi}_{x}-c_{1} I_{3} \frac{\partial \ddot{w}_{0}}{\partial x}, \\
& A_{66} \frac{\partial^{2} v_{0}}{\partial x^{2}}+A_{22} \frac{\partial^{2} v_{0}}{\partial y^{2}}+\left(A_{21}+A_{66}\right) \frac{\partial^{2} u_{0}}{\partial x \partial y}+A_{66} \frac{\partial w_{0}}{\partial y} \frac{\partial^{2} w_{0}}{\partial x^{2}}+A_{22} \frac{\partial w_{0}}{\partial y} \frac{\partial^{2} w_{0}}{\partial y^{2}}+\left(A_{21}+A_{66}\right) \frac{\partial w_{0}}{\partial x} \frac{\partial^{2} w_{0}}{\partial x \partial y}=I_{0} \ddot{v}_{0}+J_{1} \ddot{\varphi}_{y}-c_{1} I_{3} \frac{\partial \ddot{w}_{0}}{\partial y},
\end{aligned}
$$




$$
\begin{aligned}
& A_{11} \frac{\partial u_{0}}{\partial x} \frac{\partial^{2} w_{0}}{\partial x^{2}}+A_{21} \frac{\partial u_{0}}{\partial x} \frac{\partial^{2} w_{0}}{\partial y^{2}}+2 A_{66} \frac{\partial u_{0}}{\partial y} \frac{\partial^{2} w_{0}}{\partial x \partial y}+A_{11} \frac{\partial^{2} u_{0}}{\partial x^{2}} \frac{\partial w_{0}}{\partial x}+\left(A_{21}+A_{66}\right) \frac{\partial^{2} u_{0}}{\partial x \partial y} \frac{\partial w_{0}}{\partial y}+A_{66} \frac{\partial^{2} v_{0}}{\partial x^{2}} \frac{\partial w_{0}}{\partial y}+\left(A_{12}+A_{66}\right) \frac{\partial^{2} v_{0}}{\partial x \partial y} \frac{\partial w_{0}}{\partial x} \\
& \quad+A_{22} \frac{\partial^{2} v_{0}}{\partial y^{2}} \frac{\partial w_{0}}{\partial y}+\frac{3}{2} A_{11}\left(\frac{\partial w_{0}}{\partial x}\right)^{2} \frac{\partial^{2} w_{0}}{\partial x^{2}}+\left(\frac{1}{2} A_{21}+A_{66}\right)\left(\frac{\partial w_{0}}{\partial x}\right)^{2} \frac{\partial^{2} w_{0}}{\partial y^{2}}+\left(A_{12}+A_{21}+4 A_{66}\right) \frac{\partial w_{0}}{\partial x} \frac{\partial w_{0}}{\partial y} \frac{\partial^{2} w_{0}}{\partial x \partial y}+\frac{3}{2} A_{22}\left(\frac{\partial w_{0}}{\partial y}\right)^{2} \frac{\partial^{2} w_{0}}{\partial y^{2}} \\
& \quad+\left(\frac{1}{2} A_{12}+A_{66}\right)\left(\frac{\partial w_{0}}{\partial y}\right)^{2} \frac{\partial^{2} w_{0}}{\partial x^{2}}+\left(F_{0}+F_{1} \cos \Omega_{1} t\right) \frac{\partial^{2} w_{0}}{\partial y^{2}}+\left(A_{55}-2 c_{2} D_{55}+c_{2}^{2} F_{55}\right) \frac{\partial^{2} w_{0}}{\partial x^{2}}+A_{22} \frac{\partial v_{0}}{\partial y} \frac{\partial^{2} w_{0}}{\partial y^{2}}+A_{66} \frac{\partial w_{0}}{\partial x} \frac{\partial^{2} u_{0}}{\partial y^{2}} \\
& \quad+2 A_{66} \frac{\partial v_{0}}{\partial x} \frac{\partial^{2} w_{0}}{\partial x \partial y}+A_{12} \frac{\partial v_{0}}{\partial y} \frac{\partial^{2} w_{0}}{\partial x^{2}}+\left(A_{44}-2 c_{2} D_{44}+c_{2}^{2} F_{44}\right) \frac{\partial^{2} w_{0}}{\partial y^{2}}-c_{1}^{2} H_{11} \frac{\partial^{4} w_{0}}{\partial x^{4}}-c_{1}^{2}\left(H_{12}+H_{21}+4 H_{66}\right) \frac{\partial^{4} w_{0}}{\partial x^{2} \partial y^{2}} \\
& \quad+\left(A_{55}-2 c_{2} D_{55}+c_{2}^{2} F_{55}\right) \frac{\partial \varphi_{x}}{\partial x}-c_{1}^{2} H_{22} \frac{\partial^{4} w_{0}}{\partial y_{4}}+c_{1}\left(F_{11}-c_{1} H_{11}\right) \frac{\partial^{3} \varphi_{x}}{\partial x^{3}}+\left(A_{44}-2 c_{2} D_{44}+c_{2}^{2} F_{44}\right) \frac{\partial \varphi_{y}}{\partial y} \\
& +c_{1}\left(F_{21}+2 F_{66}-c_{1} H_{21}-2 c_{1} H_{66}\right) \frac{\partial^{3} \varphi_{x}}{\partial x \partial y^{2}}+c_{1}\left(F_{12}+2 F_{66}-c_{1} H_{12}-2 c_{1} H_{66}\right) \frac{\partial^{3} \varphi_{x}}{\partial x^{2} \partial y} \\
& +c_{1}\left(F_{22}-c_{1} H_{22}\right) \frac{\partial^{3} \varphi_{y}}{\partial y^{3}}+L-\gamma \dot{w}_{0}=I_{0} \ddot{w}_{0}-c_{1}^{2} I_{6}\left(\frac{\partial^{2} \ddot{w}_{0}}{\partial x^{2}}+\frac{\partial^{2} \ddot{w}_{0}}{\partial y^{2}}\right)+c_{1} I_{3}\left(\frac{\partial \ddot{u}_{0}}{\partial x}+\frac{\partial \ddot{v}_{0}}{\partial y}\right)+c_{1} J_{4}\left(\frac{\partial \ddot{\varphi}_{x}}{\partial x}+\frac{\partial \ddot{\varphi}_{y}}{\partial y}\right)
\end{aligned}
$$$$
\left(D_{11}-2 c_{1} F_{11}+c_{1}^{2} H_{11}\right) \frac{\partial^{2} \varphi_{x}}{\partial x^{2}}+\left(D_{66}-2 c_{1} F_{66}+c_{1}^{2} H_{66}\right) \frac{\partial^{2} \varphi_{x}}{\partial y^{2}}-c_{1}\left(F_{11}-c_{1} H_{11}\right) \frac{\partial^{3} w_{0}}{\partial x^{3}}
$$$$
-\left(A_{55}-2 c_{2} D_{55}+c_{2}^{2} F_{55}\right) \frac{\partial w_{0}}{\partial x}+\left(D_{12}+D_{66}+c_{1}^{2} H_{66}-2 c_{1} F_{66}+c_{1}^{2} H_{12}-2 c_{1} F_{12}\right) \frac{\partial^{2} \varphi_{y}}{\partial x \partial y}
$$$$
-c_{1}\left(F_{12}+2 F_{66}-c_{1} H_{12}-2 c_{1} H_{66}\right) \frac{\partial^{3} w_{0}}{\partial x \partial y^{2}}+\left(2 c_{2} D_{55}-A_{55}-c_{2}^{2} F_{55}\right) \varphi_{x}=J_{1} \ddot{u}_{0}+K_{2} \ddot{\varphi}_{x}-c_{1} J_{4} \frac{\partial \ddot{w}_{0}}{\partial x},
$$$$
\left(D_{66}-2 c_{1} F_{66}+c_{1}^{2} H_{66}\right) \frac{\partial^{2} \varphi_{y}}{\partial x^{2}}-c_{1}\left(F_{21}+2 F_{66}-c_{1} H_{21}-2 c_{1} H_{66}\right) \frac{\partial^{3} w_{0}}{\partial x^{2} \partial y}-c_{1}\left(F_{22}-c_{1} H_{22}\right) \frac{\partial^{3} w_{0}}{\partial y^{3}}
$$$$
+\left(D_{21}+D_{66}+c_{1}^{2} H_{21}-2 c_{1} F_{21}+c_{1}^{2} H_{66}-2 c_{1} F_{66}\right) \frac{\partial^{2} \varphi_{x}}{\partial x \partial y}+\left(c_{1}^{2} H_{22}+D_{22}-2 c_{1} F_{22}\right) \frac{\partial^{2} \varphi_{y}}{\partial x^{2}}
$$$$
-\left(F_{44} c_{2}^{2}-2 c_{2} D_{44}+A_{44}\right) \frac{\partial w_{0}}{\partial y}+\left(2 c_{2} D_{44}-A_{44}-c_{2}^{2} F_{44}\right) \varphi_{y}=J_{1} \ddot{v}_{0}+K_{2} \ddot{\varphi}_{y}-c_{1} J_{4} \frac{\partial \ddot{w}_{0}}{\partial y},
$$

where

$$
\begin{aligned}
& \left(A_{i j}, D_{i j}, F_{i j}\right)=\sum_{k=1}^{N} \int_{z_{k}}^{z_{k+1}} Q_{i j}^{k}\left(1, z^{2}, z^{4}\right) d z, \\
& I_{i}=\sum_{k=1}^{3} \int_{z_{k}}^{z_{k}+1} \rho_{k}(z)^{i} d z, \quad(i=0,1,2, \ldots, 6), \\
& J_{i}=I_{i}-c_{1} I_{i+2}, \\
& K_{2}=I_{2}-2 c_{1} I_{4}+c_{1}^{2} I_{6} .
\end{aligned}
$$$$
\left(A_{i j}, B_{i j}, D_{i j}, E_{i j}, F_{i j}, H_{i j}\right)=\sum_{k=1}^{N} \int_{z_{k}}^{z_{k+1}} Q_{i j}^{k}\left(1, z, z^{2}, z^{3}, z^{4}, z^{6}\right) \mathrm{d} z
$$$$
(i, j=1,2,6) \text {, }
$$

\section{Data Availability}

The data used to support the findings of this study are included within the article. All the pictures and quotations are based on the theoretical principles and the conditions we give in this paper.

\section{Conflicts of Interest}

The authors declare that they have no conflicts of interest.

\section{Acknowledgments}

This work was supported by the National Natural Science Foundation of China (Grant no. 11832002) and National Major Scientific Research Instrument Research Project (Grant no. 11427801). 


\section{References}

[1] M. Mehri, H. Asadi, and M. A. Kouchakzadeh, "Computationally efficient model for flow-induced instability of CNT reinforced functionally graded truncated conical curved panels subjected to axial compression," Computer Methods in Applied Mechanics and Engineering, vol. 318, pp. 957-980, 2017.

[2] C. Y. Chia, Nonlinear Analysis of Plates, McGraw-Hill International Book Company, New York, NY, USA, 1980.

[3] C.-Y. Chia, "Geometrically nonlinear behavior of composite plates: a review," Applied Mechanics Reviews, vol. 41, no. 12, pp. 439-451, 1988.

[4] K. Mehar and S. K. Panda, "Elastic bending and stress analysis of carbon nanotube-reinforced composite plate: experimental, numerical, and simulation," Advances in Polymer Technology, vol. 37, no. 6, pp. 1643-1657, 2018.

[5] T. R. Mahapatra, V. R. Kar, and S. K. Panda, "Large amplitude vibration analysis of laminated composite spherical panels under hygrothermal environment," International Journal of Structural Stability and Dynamics, vol. 16, no. 3, Article ID 1450105, 2016.

[6] T. R. Mahapatra and S. K. Panda, "Thermoelastic vibration analysis of laminated doubly curved shallow panels using non-linear FEM," Journal of Thermal Stresses, vol. 38, no. 1, pp. 39-68, 2015.

[7] T. R. Mahapatra and S. K. Panda, "Nonlinear free vibration analysis of laminated composite spherical shell panel under elevated hygrothermal environment: a micromechanical approach," Aerospace Science and Technology, vol. 49, pp. 276288, 2016.

[8] T. R. Mahapatra, V. R. Kar, and S. K. Panda, "Nonlinear free vibration analysis of laminated composite doubly curved shell panel in hygrothermal environment," Journal of Sandwich Structures \& Materials, vol. 17, no. 5, pp. 511-545, 2015.

[9] T. R. Mahapatra, K. Mehar, S. K. Panda et al., "Flexural strength of functionally graded nanotube reinforced sandwich spherical panel. IOP conference series: materials Science and Engineering," IOP Publishing, vol. 178, no. 1, Article ID 012031, 2017.

[10] K. Mehar, S. K. Panda, and T. R. Mahapatra, "Large deformation bending responses of nanotube-reinforced polymer composite panel structure: numerical and experimental analyses," Proceedings of the Institution of Mechanical Engineers, Part G: Journal of Aerospace Engineering, vol. 233, no. 5, pp. 1695-1704, 2019.

[11] K. Mehar, S. K. Panda, and T. R. Mahapatra, "Thermoelastic nonlinear frequency analysis of CNT reinforced functionally graded sandwich structure," European Journal of Mechanics-A/Solids, vol. 65, pp. 384-396, 2017.

[12] K. Mehar and S. K. Panda, "Nonlinear finite element solutions of thermoelastic flexural strength and stress values of temperature dependent graded CNT-reinforced sandwich shallow shell structure," Structural Engineering and Mechanics, vol. 67, no. 6, pp. 565-578, 2018.

[13] K. Mehar and S. K. Panda, "Thermoelastic flexural analysis of FG-CNT doubly curved shell panel," Aircraft Engineering and Aerospace Technology, vol. 90, no. 1, pp. 11-23, 2018.

[14] V. R. Kar and S. K. Panda, "Nonlinear flexural vibration of shear deformable functionally graded spherical shell panel," Steel and Composite Structures, vol. 18, no. 3, pp. 693-709, 2015.

[15] V. R. Kar and S. K. Panda, "Nonlinear free vibration of functionally graded doubly curved shear deformable panels using finite element method," Journal of Vibration and Control, vol. 22, no. 7, pp. 1935-1949, 2016.

[16] V. R. Kar and S. K. Panda, "Geometrical nonlinear free vibration analysis of FGM spherical panel under nonlinear thermal loading with TD and TID properties," Journal of Thermal Stresses, vol. 39, no. 8, pp. 942-959, 2016.

[17] L. Librescu and J. N. Reddy, "A few remarks concerning several refined theories of anisotropic composite laminated plates," International Journal of Engineering Science, vol. 27, no. 5, pp. 515-527, 1989.

[18] V. K. Singh and S. K. Panda, "Nonlinear free vibration analysis of single/doubly curved composite shallow shell panels," ThinWalled Structures, vol. 85, pp. 341-349, 2014.

[19] K. Mehar and S. K. Panda, "Geometrical nonlinear free vibration analysis of FG-CNT reinforced composite flat panel under uniform thermal field," Composite Structures, vol. 143, pp. 336-346, 2016.

[20] S. K. Panda and B. N. Singh, "Nonlinear finite element analysis of thermal post-buckling vibration of laminated composite shell panel embedded with SMA fibre," Aerospace Science and Technology, vol. 29, no. 1, pp. 47-57, 2013.

[21] K. Mehar, S. K. Panda, T. Q. Bui, and T. R. Mahapatra, "Nonlinear thermoelastic frequency analysis of functionally graded CNT-reinforced single/doubly curved shallow shell panels by FEM," Journal of Thermal Stresses, vol. 40, no. 7, pp. 899-916, 2017.

[22] K. Mehar, S. K. Panda, and T. R. Mahapatra, "Nonlinear frequency responses of functionally graded carbon nanotubereinforced sandwich curved panel under uniform temperature field," International Journal of Applied Mechanics, vol. 10, no. 3, Article ID 1850028, 2018.

[23] W. Zhang, "Global and chaotic dynamics for a parametrically excited thin plate," Journal of Sound and Vibration, vol. 239, no. 5, pp. 1013-1036, 2001.

[24] S. K. Panda and B. N. Singh, "Nonlinear free vibration of spherical shell panel using higher order shear deformation theory-a finite element approach," International Journal of Pressure Vessels and Piping, vol. 86, no. 6, pp. 373-383, 2009.

[25] S. K. Panda and B. N. Singh, "Large amplitude free vibration analysis of thermally post-buckled composite doubly curved panel using nonlinear FEM," Finite Elements in Analysis and Design, vol. 47, no. 4, pp. 378-386, 2011.

[26] S. K. Panda and B. N. Singh, "Nonlinear free vibration analysis of thermally post-buckled composite spherical shell panel," International Journal of Mechanics and Materials in Design, vol. 6, no. 2, pp. 175-188, 2010.

[27] A. K. Onkar and D. Yadav, "Forced nonlinear vibration of laminated composite plates with random material properties," Composite Structures, vol. 70, no. 3, pp. 334-342, 2005.

[28] T. Park, S.-Y. Lee, J. W. Seo, and G. Z. Voyiadjis, "Structural dynamic behavior of skew sandwich plates with laminated composite faces," Composites Part B: Engineering, vol. 39, no. 2, pp. 316-326, 2008.

[29] R.-D. Chien and C.-S. Chen, "Nonlinear vibration of laminated plates on an elastic foundation," Thin-walled Structures, vol. 44, no. 8, pp. 852-860, 2006.

[30] B. N. Singh, A. Lal, and R. Kumar, "Nonlinear bending response of laminated composite plates on nonlinear elastic foundation with uncertain system properties," Engineering Structures, vol. 30, no. 4, pp. 1101-1112, 2008.

[31] W. Zhang, X. Y. Guo, and S. K. Lai, "Research on periodic and chaotic oscillations of composite laminated plates with oneto-one internal resonance," International Journal of Nonlinear 
Sciences and Numerical Simulation, vol. 10, pp. 1567-1584, 2009.

[32] W. Zhang, Z. Yao, and M. Yao, "Periodic and chaotic dynamics of composite laminated piezoelectric rectangular plate with one-to-two internal resonance," Science in China Series E: Technological Sciences, vol. 52, no. 3, pp. 731-742, 2009.

[33] F. Alijani, M. Amabili, K. Karagiozis, and F. Bakhtiari-Nejad, "Nonlinear vibrations of functionally graded doubly curved shallow shells," Journal of Sound and Vibration, vol. 330, no. 7, pp. 1432-1454, 2011.

[34] F. Alijani, F. Bakhtiari-Nejad, and M. Amabili, "Nonlinear vibrations of FGM rectangular plates in thermal environments," Nonlinear Dynamics, vol. 66, no. 3, pp. 251-270, 2011.

[35] M. Amabili, "Nonlinear vibrations of angle-ply laminated circular cylindrical shells: skewed modes," Composite Structures, vol. 94, no. 12, pp. 3697-3709, 2012.

[36] W. Zhang, J. Chen, Y. F. Zhang, and X. D. Yang, "Continuous model and nonlinear dynamic responses of circular mesh antenna clamped at one side," Engineering Structures, vol. 151, pp. 115-135, 2017.

[37] T. Liu, W. Zhang, and J. F. Wang, "Nonlinear dynamics of composite laminated circular cylindrical shell clamped along a generatrix and with membranes at both ends," Nonlinear Dynamics, vol. 90, no. 2, pp. 1393-1417, 2017.

[38] Y. Sun, W. Zhang, and M. H. Yao, "Multi-pulse chaotic dynamics of circular mesh antenna with 1:2 internal resonance," International Journal of Applied Mechanics, vol. 9, no. 4, Article ID 1750060, 2017.

[39] W. Zhang, T. Liu, A. Xi, and Y. N. Wang, "Resonant responses and chaotic dynamics of composite laminated circular cylindrical shell with membranes," Journal of Sound and Vibration, vol. 423, pp. 65-99, 2018.

[40] E. H. Dowell, "Nonlinear oscillations of a fluttering plate," AIAA Journal, vol. 4, no. 7, pp. 1267-1275, 1966.

[41] E. H. Dowell, "Nonlinear oscillations of a fluttering plate. II," AIAA Journal, vol. 5, no. 10, pp. 1856-1862, 1967.

[42] M. Amabili and M. P. Par"doussis, "Review of studies on geometrically nonlinear vibrations and dynamics of circular cylindrical shells and panels, with and without fluid-structure interaction," Applied Mechanics Reviews, vol. 56, no. 4, pp. 349-381, 2003.

[43] M. K. Singha and M. Ganapathi, "A parametric study on supersonic flutter behavior of laminated composite skew flat panels," Composite Structures, vol. 69, no. 1, pp. 55-63, 2005.

[44] H. Haddadpour, H. M. Navazi, and F. Shadmehri, "Nonlinear oscillations of a fluttering functionally graded plate," Composite Structures, vol. 79, no. 2, pp. 242-250, 2007.

[45] M. K. Singha and M. Mandal, "Supersonic flutter characteristics of composite cylindrical panels," Composite Structures, vol. 82, no. 2, pp. 295-301, 2008.

[46] S.-Y. Kuo, "Flutter of rectangular composite plates with variable fiber pacing," Composite Structures, vol. 93, no. 10, pp. 2533-2540, 2011.

[47] M. H. Zhao and W. Zhang, "Nonlinear dynamics of composite laminated cantilever rectangular plate subject to thirdorder piston aerodynamics," Acta Mechanica, vol. 225, no. 7, pp. 1985-2004, 2014.

[48] Y. X. Hao, W. Zhang, J. Yang, and S. B. Li, "Nonlinear dynamics of a functionally graded thin simply-supported plate under a hypersonic flow," Mechanics of Advanced Materials and Structures, vol. 22, no. 8, pp. 619-632, 2015.

[49] S. F. Lu, W. Zhang, and X. J. Song, "Time-varying nonlinear dynamics of a deploying piezoelectric laminated composite plate under aerodynamic force," Acta Mechanica Sinica, vol. 34, pp. 1-12, 2017.

[50] G. Yao and F.-M. Li, "Chaotic motion of a composite laminated plate with geometric nonlinearity in subsonic flow," International Journal of Non-Linear Mechanics, vol. 50, pp. 81-90, 2013.

[51] J. Li, Y. Yan, Z. Liang, and T. Zhang, "Experimental and numerical study of adhesively bonded CFRP scarf-Lap joints subjected to tensile loads," The Journal of Adhesion, vol. 92, no. 1, pp. 1-17, 2016.

[52] A. H. Nayfeh and D. T. Mook, Nonlinear Oscillations, Oxford University Press, Oxford, UK, 1981. 